\title{
Lagrangian reduction by stages for non-holonomic systems in a Lie algebroid framework
}

\author{
Tom Mestdag ${ }^{1}$ \\ Department of Mathematical Physics and Astronomy, Ghent University, Krijgslaan 281, \\ B-9000 Ghent, Belgium \\ E-mail: Tom.Mestdag@UGent.be
}

Received 25 May 2005, in final form 19 August 2005

Published 9 November 2005

Online at stacks.iop.org/JPhysA/38/10157

\begin{abstract}
The Lagrange-d'Alembert equations of a non-holonomic system with symmetry can be reduced to the Lagrange-d'Alembert-Poincaré equations. In a previous contribution we have shown that both sets of equations fall in the category of the so-called 'Lagrangian systems on a subbundle of a Lie algebroid'. In this paper, we investigate the special case when the reduced system is again invariant under a new symmetry group (and so forth). Via Lie algebroid theory, we develop a geometric context in which successive reduction can be performed in an intrinsic way. We prove that, at each stage of the reduction, the reduced systems are part of the above mentioned category, and that the Lie algebroid structure in each new step is the quotient Lie algebroid of the previous step. We further show that that reduction in two stages is equivalent with direct reduction.
\end{abstract}

PACS numbers: $02.20 . \mathrm{Sv}, 45.20 . \mathrm{Jj}$

Mathematics Subject Classification: 17B66, 37J15, 70G45, 70H03, 70F25

\section{Introduction}

In this paper, we study the Lagrangian description of non-holonomic systems (with linear constraints) which are invariant under the action of a Lie group. It is well known that the Lagrange-d'Alembert equations ${ }^{2}$ of such systems project onto a new set of equations, the Lagrange-d'Alembert-Poincaré equations. In turn, these reduced equations could also be invariant under some action and could therefore be subjected to a second reduction. In this paper we will present a geometrical framework in which such successive reduction can be

1 Postdoctoral Fellow of the Special Research Fund of Ghent University.

2 We use the terminology of [2] and [3]. 
investigated. As such, we provide an answer to a problem which has been put forward in $[2,3]$.

A main source of inspiration is the work by Cendra et al [2], where it has been shown for unconstrained or holonomically constrained systems that repetitive reduction can be performed within the category of systems on the so-called Lagrange-Poincaré bundles. This category is indeed 'stable' under reduction by 'Lagrange-Poincaré actions' in the sense that the reduced system lives on some kind of quotient Lagrange-Poincaré bundle.

It is not difficult to see that a Lagrange-Poincaré bundle is in fact an example of a transitive Lie algebroid and that therefore all the systems of interest fall in the category of Weinstein's 'Lagrangian systems on Lie algebroids' [13] (see also [11]). The LagrangePoincaré equations, for example, which are obtained from a $G$-invariant Lagrangian can be regarded as Lagrange equations on the so-called Atiyah algebroid $T M / G$. Based on a geometric framework of Martínez [8], it has been shown in [5] that the map that projects the original equations to the reduced ones can be regarded as a Lie algebroid morphism between the Lie algebroid structures on so-called 'prolongation bundles' of $T M$ and $T M / G$.

In [2] it has been remarked that further exploration of the link between Lagrange-Poincaré bundles and Lie algebroids would be useful. With the results of [5] in mind, when looking at successive reduction, it makes perfect sense to extend the category of systems of interest to those on arbitrary Lie algebroids, which by itself can offer an alternative view on the results of [2]. We will go even one step further, however, as we will deal straightaway with the larger category of non-holonomic systems with symmetry. For that we can rely on [12], where we have developed a framework for studying Lagrangian systems on a subbundle $\mu$ of a Lie algebroid $\tau$ (see also [4]). In summary, the main purpose of this paper is to show that the category of Lagrangian-type systems on a subbundle of a Lie algebroid is stable under reduction. A geometric description of successive reduction of non-holonomic systems (as was asked for, e.g., in [3]) then follows automatically, and unconstrained systems are of course included in such an approach, when one takes the 'constrained bundle' $\mu$ to be simply the whole Lie algebroid $\tau$.

The paper is organized as follows. After some basic concepts and results, we introduce Lie algebroid actions and quotient Lie algebroids. Next, we recall the definition of a Lagrangian system on a subbundle of a Lie algebroid and show how these equations for a reducible Lagrangian project on those for the reduced Lagrangian. Finally, we show that reduction in two stages is equivalent with direct reduction. From this, symmetry reduction of the Lagrange-d'Alembert equations of a non-holonomic system in an arbitrary number of steps can be derived. We end the paper with coordinate expressions and some illustrative examples.

\section{Quotient Lie algebroids}

Some of the next definitions and results can be found in [2, 5-7]. Let $\bar{\pi}^{M}: M \rightarrow \bar{M}=M / G$ be a principal fibre bundle with structure group $G$ and (proper and free) action $\psi^{M}: G \times M \rightarrow$ $M ;(g, m) \mapsto g m$. Let $\tau: \mathrm{V} \rightarrow M$ be a vector bundle.

Definition 1. An action $\psi^{\vee}: G \times \vee \rightarrow \vee$ such that for each $g \in G$ the map $\psi_{g}^{\mathrm{V}}: \mathrm{V}_{m} \rightarrow \mathrm{V}_{g m}: \mathrm{v} \mapsto g \vee$ is an isomorphism (over $\psi_{g}^{M}$ ) and such that $\tau$ is equivariant (meaning that $\tau \circ \psi_{g}^{\vee}=\psi_{g}^{M} \circ \tau$ ) is called a vector bundle action.

The quotient $\overline{\mathrm{V}}=\mathrm{V} / G$ can be given the structure of a vector bundle over $\bar{M}$ by imposing that the map $\bar{\pi}^{\mathrm{V}}: \mathrm{V} \rightarrow \overline{\mathrm{V}}$ is a morphism of vector bundles (over $\bar{\pi}^{M}$ ). The projection $\bar{\tau}: \overline{\mathrm{V}} \rightarrow \bar{M}$, given by $\bar{\tau}([\mathrm{v}])=[\tau(\mathrm{v})]$, is a surjective submersion. We will use, in many different situations, 
the same notation [.] for equivalence classes; the meaning will always be clear from the context. It can further be shown that, for each $m \in M$, the restriction $\bar{\pi}_{m} \mathrm{~V}_{m} \mathrm{~V}_{m} \rightarrow \overline{\mathrm{V}}_{[m]}$ is a linear isomorphism and has the property $\bar{\pi}_{m}^{\mathrm{V}}=\bar{\pi}_{g m}^{\mathrm{V}} \circ \psi_{g}^{\mathrm{V}}$.

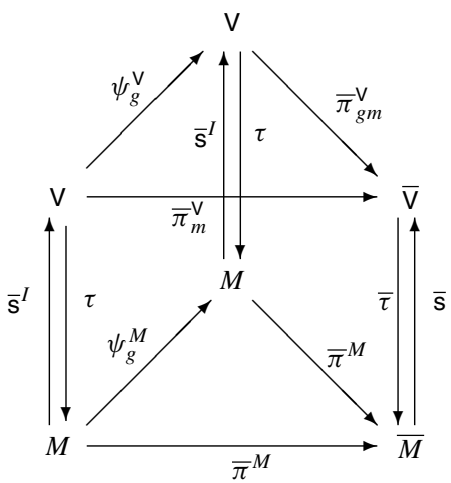

A section $r$ of $\tau$ is said to be invariant if $r(g m)=g r(m)$. The set of all invariant sections is denoted by $\operatorname{Sec}^{I}(\tau)$. Invariant sections in $\operatorname{Sec}(\tau)$ are in one-to-one correspondence with the sections of the quotient bundle $\bar{\tau}$. If $\bar{r} \in \operatorname{Sec}(\bar{\tau})$, then $\bar{r}^{I}$ will denote the corresponding invariant section of $\tau$. The diagram shows that it can in fact be defined by

$$
\overline{\mathrm{r}}^{I}(m)=\left(\bar{\pi}_{m}^{\mathrm{V}}\right)^{-1}(\overline{\mathrm{r}}([m])) .
$$

Conversely, given an invariant section $r \in \operatorname{Sec}^{I}(\tau), \mathrm{r}_{I}$ will stand for its associated section on $\bar{\tau}$, i.e.,

$$
\mathrm{r}_{I}([m])=[\mathrm{r}(m)]
$$

Likewise, invariant functions on $M$ (satisfying $f(g m)=f(m)$ ) are in one-to-one correspondence with functions on $\bar{M}$. In particular, if $\bar{f} \in C^{\infty}(\bar{M})$, then $\bar{f} \circ \bar{\pi}^{M}$ is an invariant function.

Suppose now that, in addition, $\tau: \mathrm{V} \rightarrow M$ is a Lie algebroid. Then $\operatorname{Sec}(\tau)$ is equipped with a Lie algebra bracket $[\cdot, \cdot]: \operatorname{Sec}(\tau) \times \operatorname{Sec}(\tau) \rightarrow \operatorname{Sec}(\tau)$ which is compatible with the Lie bracket of vector fields on $M$ through a linear map $\rho: \mathrm{V} \rightarrow T M$ over the identity, called the anchor map. More precisely, this compatibility means that $\forall s, r \in \operatorname{Sec}(\tau)$ and $f \in C^{\infty}(M)$

$$
[\mathbf{s}, f \mathrm{r}]=f[\mathbf{s}, \mathrm{r}]+\rho(\mathrm{s})(f) \mathrm{r},
$$

and, as a consequence, also $\rho([\mathrm{r}, \mathrm{s}])=[\rho(\mathrm{r}), \rho(\mathrm{s})]$. Notice that we will never make a notational distinction between $\rho: \mathrm{V} \rightarrow T M$ and its extension $\rho: \operatorname{Sec}(\tau) \rightarrow \mathcal{X}(M)$. In the context of vector bundles and Lie algebroids, $k$-forms on $\operatorname{Sec}(\tau)$ (from now on also called elements of $\left.\bigwedge^{k}(\tau)\right)$ are skew-symmetric, $C^{\infty}(M)$-multi-linear maps $\omega: \operatorname{Sec}(\tau) \times \cdots \times$ $\operatorname{Sec}(\tau) \rightarrow C^{\infty}(M)$ (with $k$ arguments). Let $\omega \in \bigwedge^{k}(\tau)$, then its exterior derivative is the $(k+1)$-form $\mathrm{d} \omega$, defined by

$$
\begin{aligned}
\mathrm{d} \omega\left(\mathrm{s}_{1}, \ldots, \mathrm{s}_{k+1}\right) & =\sum_{i=1}^{k+1}(-1)^{i-1} \rho\left(\mathrm{s}_{i}\right)\left(\omega\left(\mathrm{s}_{1}, \ldots, \hat{\mathrm{s}}_{i}, \ldots, \mathrm{s}_{k+1}\right)\right) \\
+ & \sum_{1 \leqslant i<j \leqslant k+1}(-1)^{i+j} \omega\left(\left[\mathrm{s}_{i}, \mathrm{~s}_{j}\right], \mathrm{s}_{1}, \ldots, \hat{\mathrm{s}}_{i}, \ldots, \hat{\mathrm{s}}_{j}, \ldots, \mathrm{s}_{k+1}\right) .
\end{aligned}
$$

In particular, on functions $\mathrm{d} f(\mathrm{r})=\rho(\mathrm{r}) f$, and on 1-forms $\mathrm{d} \theta(\mathrm{r}, \mathrm{s})=\rho(\mathrm{r})(\theta(\mathrm{s}))-\rho(\mathrm{s})(\theta(\mathrm{r}))-$ $\theta([r, s]) . d$ is a derivation of degree 1 and satisfies $d^{2}=0$. 
We will show next that, for appropriate vector bundle actions on a Lie algebroid $\tau$, the quotient bundle $\bar{\tau}$ can also be given a Lie algebroid structure. First, we need to say a few words about Lie algebroid morphisms. Let $\tau: \mathrm{V} \rightarrow M$ and $\tau^{\prime}: \mathrm{V}^{\prime} \rightarrow M^{\prime}$ be two Lie algebroids and $\Phi: \mathrm{V} \rightarrow \mathrm{V}^{\prime}$ a linear bundle map over $\phi: M \rightarrow M^{\prime}$ (i.e. a morphism of vector bundles). Then, for all $\theta^{\prime} \in \bigwedge^{k}\left(\tau^{\prime}\right), \Phi^{*} \theta^{\prime}$ given by

$$
\Phi^{*} \theta^{\prime}(m)\left(\mathrm{v}_{1}, \ldots, \mathrm{v}_{k}\right)=\theta^{\prime}(\phi(m))\left(\Phi\left(\mathrm{v}_{1}\right), \ldots \Phi\left(\mathrm{v}_{k}\right)\right), \quad \mathrm{v}_{i} \in \mathrm{V}_{m},
$$

defines a $k$-form on $\operatorname{Sec}(\tau)$. $\Phi$ is called a Lie algebroid morphism if

$$
d\left(\Phi^{*} \theta^{\prime}\right)=\Phi^{*}\left(d^{\prime} \theta^{\prime}\right)
$$

for all $\theta^{\prime} \in \bigwedge\left(\tau^{\prime}\right)$. In fact, by the derivation property, it suffices that the above relation is satisfied for functions and 1-forms. A map $\Phi$ satisfying (5) only for functions is said to be admissible. Equivalently, admissible maps can be characterized by the condition

$$
T \phi \circ \rho=\rho^{\prime} \circ \Phi \text {. }
$$

When $\Phi$ is fibrewise surjective, condition (5) on 1-forms can be recast in the following form: if $r_{i}$ and $r_{i}^{\prime}$ are $\Phi$-related sections of, respectively, $\tau$ and $\tau^{\prime}$, then also $\left[r_{1}, r_{2}\right]$ is $\Phi$-related with $\left[r_{1}^{\prime}, r_{2}^{\prime}\right]$ (see [6]). When, in addition, $\phi: M \rightarrow M^{\prime}$ is a diffeomorphism, there is a more direct way to characterize the preservation of the Lie algebroid structure. Indeed, now $\Phi_{*}(r) \in \operatorname{Sec}\left(\tau^{\prime}\right)$ can be defined by

$$
\Phi_{*}(r)\left(m^{\prime}\right)=\Phi\left(r\left(\phi^{-1}(m)\right)\right)
$$

(for $r \in \operatorname{Sec}(\tau)$ ) and the requirement (5) for functions and 1-forms becomes

$$
\rho^{\prime}\left(\Phi_{*}(\mathrm{r})\right)=\phi_{*}(\rho(\mathrm{r})) \quad \text { and } \quad \Phi_{*}([\mathrm{r}, \mathrm{s}])=\left[\Phi_{*}(\mathrm{r}), \Phi_{*}(\mathrm{~s})\right],
$$

where $\phi_{*} X$ stands for the push-forward of the vector field $X$ on $M$. So, if we want to check that an admissible $\Phi$ is a Lie algebroid morphism, we only need to check that $\Phi_{*}$ is a Lie algebra morphism between the (real) Lie algebras $\operatorname{Sec}(\tau)$ and $\operatorname{Sec}\left(\tau^{\prime}\right)$. An important case is when $M=M^{\prime}$ and $\phi=$ id. In such a case, we will simply write $\Phi(r)$ for $\Phi_{*}(r)$.

Definition 2. A vector bundle action $\psi^{\vee}$ is a Lie algebroid action if $\psi_{g}^{\vee}$ is a Lie algebroid isomorphism over $\psi_{g}^{M}$ for all $g \in G$.

In what follows we will always assume that the action $\psi^{\vee}$ is a Lie algebroid action. As a consequence, for each fixed $g$,

$$
\left(\psi_{g}^{\mathrm{V}}\right)_{*}([\mathrm{r}, \mathrm{s}])=\left[\left(\psi_{g}^{\mathrm{V}}\right)_{*}(\mathrm{r}),\left(\psi_{g}^{\mathrm{V}}\right)_{*}(\mathrm{~s})\right] .
$$

An invariant section $r \in \operatorname{Sec}^{I}(\tau)$ satisfies $\left(\psi_{g}^{\vee}\right)_{*}(r)=r$ and is thus always, for each $g \in G, \psi_{g}^{\vee}$ related to itself. As a result, the set of invariant sections forms a Lie subalgebra (of the (real) Lie algebra of sections), and since invariant sections can be identified with sections of the quotient bundle $\bar{\tau}: \bar{V} \rightarrow \bar{M}$, this quotient bundle inherits a Lie algebroid structure. Indeed, for a start, the Lie bracket of the two sections $\bar{r}$ and $\bar{s}$ of $\bar{\tau}$ can be defined by

$$
[\overline{\mathrm{r}}, \overline{\mathrm{s}}]=\left(\left[\overline{\mathrm{r}}^{I}, \overline{\mathrm{s}}^{I}\right]\right)_{I} \text {. }
$$

Next, an appropriate anchor map can be defined as follows: since the anchor map $\rho$ is equivariant by assumption, i.e. $\rho \circ \psi_{g}^{\vee}=T \psi_{g}^{M} \circ \rho$, the map $\bar{\rho}: \bar{V} \rightarrow T \bar{M}$, given by the relation

$$
\bar{\rho}[\mathrm{v}]=T \bar{\pi}^{M}(\rho(\mathrm{v}))
$$

is well defined. At the level of sections it has the property

$$
(\bar{\rho}(\overline{\mathrm{r}}) \bar{f}) \circ \bar{\pi}^{M}=\rho\left(\overline{\mathrm{r}}^{I}\right)\left(\bar{f} \circ \bar{\pi}^{M}\right), \quad \forall \bar{f} \in C^{\infty}(M)
$$


and can therefore be used as an anchor map for the quotient Lie algebroid. Indeed, it is easy to see that $(\bar{f} \overline{\mathrm{r}})^{I}=\left(\bar{f} \circ \bar{\pi}^{M}\right) \overline{\mathrm{r}}^{I}$ and therefore

$$
\begin{aligned}
{[\bar{f} \overline{\mathrm{r}}, \overline{\mathrm{s}}] } & =\left(\left[\left(\bar{f} \circ \bar{\pi}^{M}\right) \overline{\mathrm{r}}^{I}, \overline{\mathrm{s}}^{I}\right]\right)_{I}=\left(\left(\bar{f} \circ \bar{\pi}^{M}\right)\left[\overline{\mathrm{r}}^{I}, \overline{\mathrm{s}}^{I}\right]\right)_{I}-\left(\rho\left(\overline{\mathrm{s}}^{I}\right)\left(\bar{f} \circ \bar{\pi}^{M}\right) \overline{\mathrm{r}}^{I}\right)_{I} \\
& =\bar{f}[\overline{\mathrm{r}}, \overline{\mathrm{s}}]-\left((\bar{\rho}(\overline{\mathrm{s}}) \bar{f}) \circ \bar{\pi}^{M} \overline{\mathrm{r}}^{I}\right)_{I}=\bar{f}[\overline{\mathrm{r}}, \overline{\mathrm{s}}]-(\bar{\rho}(\overline{\mathrm{s}}) \bar{f}) \overline{\mathrm{r}},
\end{aligned}
$$

which shows that $\bar{\rho}$ is compatible with the Lie bracket. To conclude:

Lemma 1. For a Lie algebroid action $\psi^{\mathrm{V}}$, $\operatorname{Sec}^{I}(\tau)$ is a Lie subalgebra and the reduction by the group $G$ yields a Lie algebroid structure on the quotient $\bar{\tau}$ with bracket (7) and anchor (8).

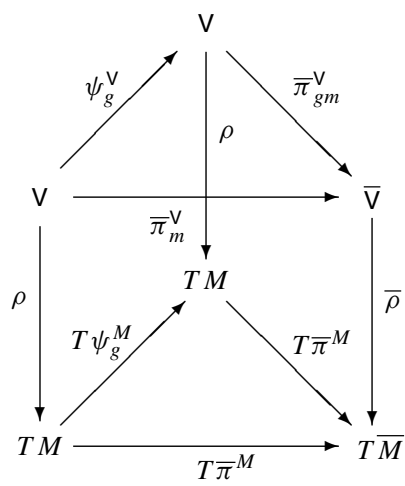

With this Lie algebroid structure on $\bar{\tau}$, the projection $\bar{\pi}^{\mathrm{V}}: \mathrm{V} \rightarrow \overline{\mathrm{V}}$ is a Lie algebroid morphism. Indeed, (8) is in fact saying that $\bar{\pi} \vee$ is admissible. Secondly, for sections $\overline{\mathrm{r}}, \overline{\mathrm{s}} \in \operatorname{Sec}(\bar{\tau})$ (that are $\bar{\pi}^{\mathrm{V}}$-related to $\overline{\mathrm{r}}^{I}$ and $\overline{\mathrm{s}}^{I}$ ), the definition of the bracket (7) shows that $[\overline{\mathrm{r}}, \overline{\mathrm{s}}]$ is $\bar{\pi}^{\mathrm{V}}$-related to $\left[\overline{\mathrm{r}}^{I}, \overline{\mathrm{s}}^{I}\right]$. Finally, we would like to remark that it is now also obvious that the $\operatorname{sets~} \operatorname{Sec}^{I}(\tau)$ and $\operatorname{Sec}(\bar{\tau})$ are isomorphic as Lie algebras.

The most simple example of a Lie algebroid is the tangent bundle with its natural bracket of vector fields and trivial anchor map. Each action $\psi^{M}$ on $M$ induces a 'tangent' action on $\mathrm{V}=T M$, given by $\psi_{g}^{T M}: T M \rightarrow T M,(m, v) \mapsto\left(g m, g v=T_{m} \psi_{g}^{M}(v)\right)$. From this definition, it is immediately clear that $\psi_{g}^{T M}$ is admissible (w.r.t. the anchor map $\rho=\mathrm{id}$ ). Further, a standard property implies that, if $X_{i}$ and $Y_{i}$ are $\psi_{g}^{\mathrm{V}}$-related vector fields, then also the vector fields $\left[X_{1}, X_{2}\right]$ and $\left[Y_{1}, Y_{2}\right]$ must be. So, $\psi^{T M}$ is a Lie algebroid action. The bracket on sections of the quotient Lie algebroid structure on $T M / G \rightarrow M / G$ (called the Atiyah algebroid) can be obtained from the Lie subalgebra of invariant vector fields, while the anchor $\operatorname{map} \bar{\rho}: T M / G \rightarrow T(M / G)$ is simply

$$
\bar{\rho}([v])=T \bar{\pi}^{M}(v) .
$$

In other terms, this relation shows that for invariant vector fields $X=\bar{Y}^{I} \in \mathcal{X}(M)$ and invariant functions $f=\bar{f} \circ \bar{\pi}^{M} \in C^{\infty}(M)$,

$$
X(f)=\bar{Y}(\bar{f}) \circ \bar{\pi}^{M} .
$$

\section{Prolongation bundles and non-holonomic systems on Lie algebroids}

First, we very briefly recall the definition of a prolongation bundle $\mu^{\rho}: T^{\rho} \mathbf{W} \rightarrow \mathrm{W}$. Let $\tau: \mathrm{V} \rightarrow M$ be a vector bundle with the anchor map $\rho: \mathrm{V} \rightarrow T M$ (at this stage $\tau$ need not necessarily be a Lie algebroid) and suppose that $\mu: \mathrm{W} \rightarrow M$ is a second vector bundle. Then 
the elements of the manifold $T^{\rho} \mathrm{W}$ are pairs $\left(\mathrm{v}, X_{\mathrm{w}}\right) \in \mathrm{V}_{m} \times T_{\mathrm{w}} \mathrm{W}$ for which $\rho(\mathrm{v})=T \mu\left(X_{\mathrm{w}}\right)$ (and therefore also $\tau(\mathrm{v})=\mu(\mathrm{w})=m)$. The projection $\mu^{\rho}$ is then nothing but $\mu^{\rho}\left(\mathrm{v}, X_{\mathrm{w}}\right)=\mathrm{w}$.

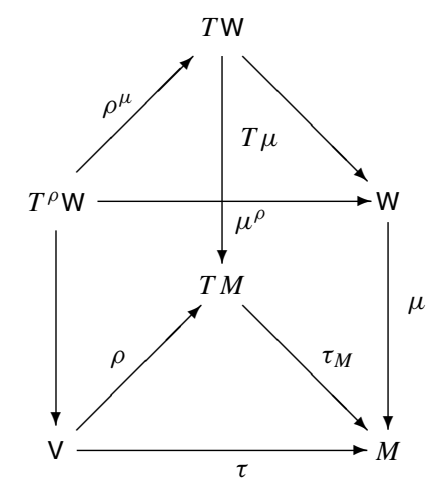

If $\tau$ is a Lie algebroid, then so is $\mu^{\rho}$ : its anchor map is simply

$$
\rho^{\mu}: T^{\rho} \mathrm{W} \rightarrow T \mathrm{~W},\left(\mathrm{v}, X_{\mathrm{w}}\right) \mapsto X_{\mathrm{w}},
$$

while its bracket can most conveniently be defined on the subset $\operatorname{Sec}^{P}\left(\mu^{\rho}\right)$ of projectable sections. These are sections of $\mu^{\rho}$ that project on a section of $\tau$, i.e. they are of the form $\mathcal{Z}=(r, X) \in \operatorname{Sec}^{P}\left(\mu^{\rho}\right)$, where $r \in \operatorname{Sec}(\tau)$, regarded as subset of $\operatorname{Sec}\left(\mu^{*} \tau\right)$. Let now $\mathcal{Z}_{i}=\left(\mathrm{r}_{i}, X_{i}\right) \in \operatorname{Sec}^{P}\left(\mu^{\rho}\right)$, then the bracket of two projectable sections is defined as

$$
\left[\mathcal{Z}_{1}, \mathcal{Z}_{2}\right]=\left(\left[\mathrm{r}_{1}, \mathrm{r}_{2}\right],\left[X_{1}, X_{2}\right]\right)
$$

Since projectable sections generate (over $C^{\infty}(\mathrm{W})$ ) all sections of $\operatorname{Sec}\left(\mu^{\rho}\right)$, the bracket of two arbitrary sections can be obtained by extending the definition (11) by means of a Leibniztype property (3) for the anchor map $\rho^{\mu}$ (for more details on prolongation Lie algebroids, see $[5,10])$.

There exists in this context a generalization of the concept tangent map. Let $\Phi: \mathrm{V}^{1} \rightarrow \mathrm{V}^{2}$ be an admissible map over $\phi: M^{1} \rightarrow M^{2}$ (between two Lie algebroids $\tau^{i}: \mathrm{V}^{i} \rightarrow M^{i}$ ) and suppose that $\Psi: \mathrm{W}^{1} \rightarrow \mathrm{W}^{2}$ is a bundle map over $\phi$ (between two arbitrary vector bundles $\left.\mu^{i}: \mathrm{W}^{i} \rightarrow M^{i}\right)$. Then, the relation

$$
\mathcal{T}^{\Phi} \Psi\left(\mathrm{v}^{1}, X_{\mathrm{w}^{1}}^{1}\right)=\left(\Phi\left(\mathrm{v}^{1}\right), T \Psi\left(X_{\mathrm{w}^{1}}^{1}\right)\right)
$$

defines a linear bundle map $\mathcal{T}^{\Phi} \Psi: T^{\rho^{1}} \mathrm{~W}^{1} \rightarrow T^{\rho^{2}} \mathrm{~W}^{2}$ over $\Psi . \mathcal{T}^{\Phi} \Psi$ is admissible with respect to the anchors $\left(\rho^{1}\right)^{\mu^{1}}$ and $\left(\rho^{2}\right)^{\mu^{2}}$ : indeed, $\left(\rho^{2}\right)^{\mu^{2}}\left(\Phi\left(\mathrm{v}^{1}\right), T \Psi\left(X_{\mathrm{w}^{1}}^{1}\right)\right)=T \Psi\left(X_{\mathrm{w}^{1}}^{1}\right)=$ $T \Psi\left(\left(\rho^{1}\right)^{\mu^{1}}\left(\mathrm{v}^{1}, X_{\mathrm{w}^{1}}^{1}\right)\right)$. It is further easy to see that if the two maps $\Phi^{i}: \mathrm{V}^{i} \rightarrow \mathrm{V}^{i+1}$ are admissible, then so is $\Phi^{2} \circ \Phi^{1}: \mathrm{V}^{1} \rightarrow \mathrm{V}^{3}$ and

$$
\mathcal{T}^{\Phi^{2} \circ \Phi^{1}}\left(\Psi^{2} \circ \Psi^{1}\right)=\mathcal{T}^{\Phi^{2}} \Psi^{2} \circ \mathcal{T}^{\Phi^{1}} \Psi^{1} .
$$

Proposition 1. $\mathcal{T}^{\Phi} \Psi$ is a Lie algebroid morphism (w.r.t. the prolonged Lie algebroid structures) if and only if $\Phi$ is a Lie algebroid morphism.

Proof. The admissibility condition on the anchor maps has already been verified. We show now that the bracket of two $\mathcal{T}^{\Phi} \Psi$-projectable sections is also $\mathcal{T}^{\Phi} \Psi$-projectable. Sections $\mathcal{Z}_{a, b}^{2}=\left(\mathrm{s}_{a, b}^{2}, X_{a, b}^{2}\right)$ in $\operatorname{Sec}^{P}\left(\left(\mu^{2}\right)^{\rho^{2}}\right)$ that are $\mathcal{T}^{\Phi} \Psi$-related to sections $\mathcal{Z}_{a, b}^{1}=\left(\mathrm{s}_{a, b}^{1}, X_{a, b}^{1}\right)$ in $\operatorname{Sec}^{P}\left(\left(\mu^{1}\right)^{\rho^{1}}\right)$ satisfy this condition, if and only if $\left[\mathrm{s}_{a}^{2}, \mathrm{~s}_{b}^{2}\right]$ is $\Phi$-related to $\left[\mathrm{s}_{a}^{1}, \mathrm{~s}_{b}^{1}\right]$, or, equivalently, if and only if $\Phi$ is a Lie algebroid morphism. Observe now that $\mathcal{T}^{\Phi} \Psi$ projectable sections in $\operatorname{Sec}^{P}\left(\left(\mu^{2}\right)^{\rho^{2}}\right)$ finitely generate (over $\left.C^{\infty}\left(\mathrm{W}^{2}\right)\right)$ the set of all sections in $\operatorname{Sec}\left(\left(\mu^{2}\right)^{\rho^{2}}\right)$ that can be $\mathcal{T}^{\Phi} \Psi$-related to some section in $\operatorname{Sec}\left(\left(\mu^{1}\right)^{\rho^{1}}\right)$ : if $f^{2} \in C^{\infty}\left(\mathrm{W}^{2}\right)$ 
and if $\mathcal{Z}_{b}^{2} \in \operatorname{Sec}^{P}\left(\left(\mu^{2}\right)^{\rho^{2}}\right)$ is $\mathcal{T}^{\Phi} \Psi$-related to $\mathcal{Z}_{b}^{1} \in \operatorname{Sec}^{P}\left(\left(\mu^{1}\right)^{\rho^{1}}\right)$, then $f^{2} \mathcal{Z}_{b}^{2}$ is $\mathcal{T}^{\Phi} \Psi$ related to $\left(f^{2} \circ \Psi\right) \mathcal{Z}_{b}^{1}$. We therefore only need to show (of course under the assumption that $\Phi$ is a Lie algebroid morphism) that, for such sections, also the brackets $\left[\mathcal{Z}_{a}^{2}, f^{2} \mathcal{Z}_{b}^{2}\right]$ and $\left[\mathcal{Z}_{a}^{1},\left(f^{2} \circ \Psi\right) \mathcal{Z}_{b}^{1}\right]$ are $\mathcal{T}^{\Phi} \Psi$-related. From the Leibniz-identity of the bracket it follows that $\left[\mathcal{Z}_{a}^{2}, f^{2} \mathcal{Z}_{b}^{2}\right]\left(\Psi\left(\mathrm{w}^{1}\right)\right)=f^{2}\left(\Psi\left(\mathrm{w}^{1}\right)\right)\left[\mathcal{Z}_{a}^{2}, \mathcal{Z}_{b}^{2}\right]\left(\Psi\left(\mathrm{w}^{1}\right)\right)+\left(\left(\rho^{2}\right)^{\mu^{2}}\left(\mathcal{Z}_{a}^{2}\right) f^{2}\right)\left(\Psi\left(\mathrm{w}^{1}\right)\right) \mathcal{Z}_{b}^{2}\left(\Psi\left(\mathrm{w}^{1}\right)\right)$.

On the one hand $\mathcal{Z}_{a}^{2}\left(\Psi\left(\mathrm{w}^{1}\right)\right)=\left(\mathrm{s}_{a}^{2}\left(\phi\left(m^{1}\right), X_{a}^{2}\left(\Psi\left(\mathrm{w}^{1}\right)\right)\right)\right)$, while, due to the supposed $\mathcal{T}^{\Phi} \Psi$-projectability, also $\mathcal{Z}_{a}^{2}\left(\Psi\left(\mathrm{w}^{1}\right)\right)=\mathcal{T}^{\Phi} \Psi\left(\mathcal{Z}_{a}^{1}\left(\mathrm{w}^{1}\right)\right)=\left(\Phi\left(\mathrm{s}_{a}^{1}\left(m^{1}\right)\right), T \Psi\left(X_{a}^{1}\left(\mathrm{w}^{1}\right)\right)\right)$. So, $X_{a}^{2}=\left(\rho^{2}\right)^{\mu^{2}}\left(\mathcal{Z}_{a}^{2}\right) \in \mathcal{X}\left(\mathbf{W}^{2}\right)$ is $T \Psi$-related to $X_{a}^{1}=\left(\rho^{1}\right)^{\mu^{1}}\left(\mathcal{Z}_{a}^{1}\right) \in \mathcal{X}\left(\mathbf{W}^{1}\right)$. Applying this property to functions $f^{2} \in C^{\infty}\left(\mathrm{W}^{2}\right)$, we get that $\left(\left(\rho^{2}\right)^{\mu^{2}}\left(\mathcal{Z}_{a}^{2}\right) f^{2}\right) \circ \Psi=\left(\rho^{1}\right)^{\mu^{1}}\left(\mathcal{Z}_{a}^{1}\right)\left(f^{2} \circ \Psi\right)$. Relation (14) now becomes

$$
\begin{aligned}
{\left[\mathcal{Z}_{a}^{2}, f^{2} \mathcal{Z}_{b}^{2}\right]\left(\Psi\left(\mathrm{w}^{1}\right)\right) } & =\mathcal{T}^{\Phi} \Psi\left(\left(f^{2} \circ \Psi\right)\left[\mathcal{Z}_{a}^{1}, \mathcal{Z}_{b}^{1}\right]+\left(\rho^{1}\right)^{\mu^{1}}\left(\mathcal{Z}_{a}^{1}\right)\left(f^{2} \circ \Psi\right) \mathcal{Z}_{b}^{1}\right)\left(\mathrm{w}^{1}\right) \\
& =\mathcal{T}^{\Phi} \Psi\left(\left[\mathcal{Z}_{a}^{1},\left(f^{2} \circ \Psi\right) \mathcal{Z}_{b}^{1}\right]\right)\left(\mathrm{w}^{1}\right),
\end{aligned}
$$

which concludes the proof.

A different proof for the above property can be found in [9], proposition 1.

We will recall now in a nutshell our definition of a Lagrangian system on a (vector) sub-bundle $\mu: \mathrm{W} \rightarrow M$ of a Lie algebroid $\tau: \mathrm{V} \rightarrow M$ (see [12]). Denote the injection $\mathrm{W} \rightarrow \mathrm{V}$ by $i$ and put

$$
\lambda=\rho \circ i \text {. }
$$

Both the prolongations $\tau^{\rho}: T^{\rho} \mathrm{V} \rightarrow \mathrm{V}$ and $\mu^{\rho}: T^{\rho} \mathrm{W} \rightarrow \mathrm{W}$ are Lie algebroids. In fact, via the injection

$$
\mathcal{T}^{i d} i: T^{\rho} \mathrm{W} \rightarrow T^{\rho} \mathrm{V}, \quad\left(\mathrm{v}, X_{\mathrm{w}}\right) \mapsto\left(\mathrm{v}, T i\left(X_{\mathrm{w}}\right)\right)
$$

it can be proved that $\mu^{\rho}$ is a Lie subalgebroid of $\tau^{\rho}$. Although $\mu$ is not a Lie (sub)algebroid, $\lambda$ will play the role of its 'anchor'. The vector bundle $\mu^{\lambda}: T^{\lambda} \mathrm{W} \rightarrow \mathrm{W}$ is a subbundle of $\mu^{\rho}$ whose injection is given by

$$
\mathcal{T}^{i} \mathrm{id}: T^{\lambda} \mathrm{W} \rightarrow T^{\rho} \mathrm{W}, \quad\left(\mathrm{w}_{1}, X_{\mathrm{w}_{2}}\right) \mapsto\left(i\left(\mathrm{w}_{1}\right), X_{\mathrm{w}_{2}}\right) .
$$

Note that $\mathcal{T}^{i}$ id is well defined because (15) can be interpreted as saying that $i$ is admissible with respect to the anchors $\rho$ and $\lambda$. In [12], $\mathcal{T}^{i}$ id and $\mathcal{T}^{\text {id }} i$ were denoted respectively by $I$ and $T^{\rho} i$. Their composition is the injection $\mathcal{T}^{i} i$, which can be used to interpret $\mu^{\lambda}$ also as a subbundle of $\tau^{\rho}$.

We need to define some canonical objects on $\tau^{\rho}$. The vertical lift ${ }^{V}: \tau^{*} \mathrm{~V} \rightarrow T^{\rho} \mathrm{V}$ is given by $(\mathrm{a}, \mathrm{v}) \in \tau^{*} \mathrm{~V} \mapsto\left(0, X_{\mathrm{a}}\right) \in T^{\rho} \mathrm{V}$, where, on a function $f \in C^{\infty}(\mathrm{V})$,

$$
X_{\mathrm{a}}(f)=\left.\frac{\mathrm{d}}{\mathrm{d} t} f(\mathrm{a}+t \mathrm{v})\right|_{t=0} .
$$

We will also use the so-called vertical endomorphism $S^{\tau}={ }^{V} \circ j: \operatorname{Sec}\left(\tau^{\rho}\right) \rightarrow \operatorname{Sec}\left(\tau^{\rho}\right)$. Here $j$ stands for the projection $\left(\mathrm{v}_{2}, X_{\mathrm{v}_{1}}\right) \in T^{\rho} \mathrm{V} \mapsto\left(\mathrm{v}_{1}, \mathrm{v}_{2}\right) \in \tau^{*} \mathrm{~V}$. Next, the Liouville section $\mathcal{C}^{\tau} \in \operatorname{Sec}\left(\tau^{\rho}\right)$ can be defined as the map $\mathrm{v} \in \mathrm{V} \mapsto(\mathrm{v}, \mathrm{v})^{V} \in T^{\rho} \mathrm{V}$. Finally, we will need the exterior derivative $\tilde{\mathrm{d}}: \bigwedge^{k}\left(\mu^{\rho}\right) \rightarrow \bigwedge^{k+1}\left(\mu^{\rho}\right)$ of the Lie algebroid $\mu^{\rho}$ to define an operator

$$
\delta=\left(\mathcal{T}^{i} \mathrm{id}\right)^{*} \circ \tilde{\mathrm{d}}: \bigwedge^{k}\left(\mu^{\rho}\right) \rightarrow \bigwedge^{k+1}\left(\mu^{\lambda}\right) .
$$


Let $L \in C^{\infty}(\mathrm{V})$ be a Lagrangian, $\theta_{L}=S^{\tau}(\mathrm{d} L) \in \bigwedge^{1}\left(\tau^{\rho}\right)$ and $E_{L}=\rho^{\tau}\left(\mathcal{C}^{\tau}\right) L-L \in$ $C^{\infty}(\mathrm{V})$. Then, we can define the Poincaré-Cartan 1-form $\tilde{\theta}_{L}$ as a 1-form on $\operatorname{Sec}\left(\mu^{\rho}\right)$ by means of $\tilde{\theta}_{L}=\left(\mathcal{T}^{\mathrm{id}} i\right)^{*} \theta_{L}$. The function $\tilde{E}_{L} \in C^{\infty}(\mathrm{W})$ is the restriction of $E_{L}$ to W. From now on, we will only consider regular Lagrangians, i.e., those for which the two-form $\delta \tilde{\theta}_{L} \in \bigwedge^{2}\left(\mu^{\lambda}\right)$ is non-degenerate.

Definition 3. [12] If $\Gamma$ is the section of the prolongation bundle $\mu^{\lambda}$, determined by

$$
i_{\Gamma} \delta \tilde{\theta}_{L}=-\delta \tilde{E}_{L},
$$

the vector field $\lambda^{\mu}(\Gamma) \in \mathcal{X}(\mathrm{W})$ is said to define the Lagrangian system on the subbundle $\mu$ of the Lie algebroid $\tau$, associated with the given Lagrangian $L$ on $\mathrm{V}$.

Another way of writing (19) is the following.

Proposition 2. Let $\mathrm{d}: \bigwedge^{k}\left(\tau^{\rho}\right) \rightarrow \bigwedge^{k+1}\left(\tau^{\rho}\right)$ be the exterior derivative of the Lie algebroid $\tau^{\rho}$ and put

$$
\Delta=\left(\mathcal{T}^{i} i\right)^{*} \circ \mathrm{d}: \bigwedge\left(\tau^{\rho}\right) \rightarrow \bigwedge\left(\mu^{\lambda}\right)
$$

then the Lagrangian section $\Gamma$ is a solution of

$$
i_{\Gamma} \Delta \theta_{L}=-\Delta E_{L} .
$$

Proof. Since $\mathcal{T}^{\mathrm{id}} i$ is a Lie algebroid morphism, $\tilde{\mathrm{d}} \circ\left(\mathcal{T}^{\mathrm{id}} i\right)^{*}=\left(\mathcal{T}^{\mathrm{id}} i\right)^{*} \circ \mathrm{d}$. It follows that $\delta \tilde{\theta}_{L}=\left(\mathcal{T}^{i} \mathrm{id}\right)^{*} \circ \tilde{\mathrm{d}} \circ\left(\mathcal{T}^{\mathrm{id}} i\right)^{*} \theta_{L}=\left(\mathcal{T}^{i} \mathrm{id}\right)^{*} \circ\left(\mathcal{T}^{\mathrm{id}} i\right)^{*} \circ \mathrm{d} \theta_{L}=\left(\mathcal{T}^{i} i\right)^{*} \circ \mathrm{d} \theta_{L}=\Delta \theta_{L}$. A similar reasoning shows that $\delta \tilde{E}_{L}=\Delta E_{L}$.

Coordinate expressions for such dynamical systems can be found in [12], where we have also shown that, in case the Lie algebroid is the standard one, i.e. $\mathrm{V}=T M$, and the subbundle $\mu$ is a distribution $\mathrm{W} \subset T M$, the above equations are exactly the Lagranged'Alembert equations for systems subject to (non-holonomic) linear constraints (see e.g. [1, p 217] for some coordinate expressions). It is further well known that, when the Lagrangian is invariant under the action of a Lie group $G$, and when the distribution satisfies some additional requirements, the Lagrange-d'Alembert equations can be reduced to the so-called Lagranged'Alembert-Poincaré equations (see e.g. [3] or [1, p 269]). We have shown in [12] that also this type of Lagrangian equations fit in our current framework, simply by considering the reduced Lagrangian on the Atiyah algebroid $\tau: T M / G \rightarrow M / G$ and by assuming that the subbundle $\mu$ is the quotient $\mathrm{W} / G \rightarrow M / G$ of the distribution.

Equation (21) (or (19)) can be simplified in the case that we are dealing with 'unconstrained' systems. Indeed, in such a case the subbundle $\mu$ is the whole Lie algebroid $\tau$ and the operators $\delta$ and $\Delta$ both equal the exterior derivative $\mathrm{d}$ on $\tau^{\rho}$. The equation for Lagrangian systems on a Lie algebroid is then simply (see [8])

$$
i_{\Gamma} \mathrm{d} \theta_{L}=-\mathrm{d} E_{L} .
$$

In the case of the standard Lie algebroid $\mathrm{V}=T M$, (22) defines the standard Euler-Lagrange equations. In the case of an (unconstrained) system with symmetry with reduced Lagrangian $L \in C^{\infty}(\mathrm{V}=T M / G)$, local expressions for equation (22) of a Lagrangian system on the Atiyah algebroid $T M / G$ are the so-called Lagrange-Poincaré equations (see also [11]). In [5] it has been proved that one can go from the system on $T M$ to the one of $T M / G$ by means of a Lie algebroid morphism.

The main purpose of this paper is to extend the results of [5] in two ways, first by allowing constraints, and secondly by taking the Lagrangian systems on an arbitrary Lie algebroid as the 
starting point for a symmetry reduction, rather than the standard Lagrangian systems. Then, a framework for repetitive reduction will follow almost automatically. Indeed, in the second step of the reduction, for example, the system on $T M / G$ is reduced to a system on a quotient $(T M / G) / H$. Within our approach, this will be interpreted as a reduction from a Lie algebroid to its quotient Lie algebroid. Of course, a lot of proofs in the next sections are inspired by those in [5].

\section{Quotients of prolongation bundles}

Definition 4. A constrained Lie algebroid action is a vector bundle action $\psi^{\mathrm{W}}: G \times \mathrm{W} \rightarrow \mathrm{W}$ (over $\psi^{M}$ ) on a subbundle $\mu$ of a Lie algebroid $\tau$ which is the restriction of a Lie algebroid action $\psi^{\mathrm{V}}: G \times \mathrm{V} \rightarrow \mathrm{V}\left(\right.$ also over $\left.\psi^{M}\right)$ to $\mathrm{W}$.

Then, $\psi_{g}^{\mathrm{V}} \circ i=i \circ \psi_{g}^{\mathrm{W}}$, from which it follows that the map $\bar{i}: \overline{\mathrm{W}} \rightarrow \overline{\mathrm{V}},[\mathrm{w}] \mapsto[i(\mathrm{w})]$ is a well-defined injection, and therefore $\bar{\mu}: \overline{\mathrm{W}} \rightarrow \bar{M}$ is a vector subbundle of $\bar{\tau}: \overline{\mathrm{V}} \rightarrow \bar{M}$. Another way of writing the definition of $\bar{i}$ is

$$
\bar{i} \circ \bar{\pi}^{\mathrm{W}}=\bar{\pi}^{\mathrm{V}} \circ i
$$

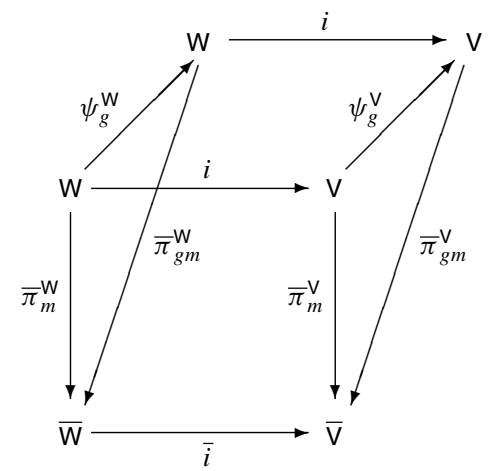

We can now define also a $G$-action on the prolongation $\mu^{\rho}$. It is given by

$$
G \times T^{\rho} \mathrm{W} \rightarrow T^{\rho} \mathrm{W}:\left(g,\left(\mathrm{v}, X_{\mathrm{w}}\right)\right) \mapsto\left(g \vee, g X_{\mathrm{w}}\right)
$$

The action in the second argument is $\psi^{T \mathrm{~W}}$, the tangent lift of the action on the manifold W. Remark that $\left(g \mathrm{v}, g X_{\mathrm{W}}\right)$ is indeed an element of $T^{\rho} \mathrm{W}$ since $\rho(g \mathrm{v})=g \rho(\mathrm{v})=g T \mu\left(X_{\mathrm{W}}\right)=$ $T \mu\left(g X_{\mathrm{w}}\right)$ ( $\rho$ by the assumption that $\psi^{\vee}$ is a Lie algebroid action, and $T \mu$ by the property $\psi_{g}^{M} \circ \mu=\mu \circ \psi_{g}^{\mathrm{W}}$, are equivariant under the appropriate actions). In fact, for a fixed $g \in G$, the above action can be rewritten as $\mathcal{T}_{g}^{\mathrm{V}} \psi_{g}^{\mathrm{W}}$. Since any $\psi_{g}^{\mathrm{V}}$ is supposed to be a Lie algebroid morphism, $\mathcal{T}^{\psi_{g}^{\mathrm{v}}} \psi_{g}^{\mathrm{W}}$ must also be a Lie algebroid morphism (for the Lie algebroid structure on the prolongation bundle). The action on $T^{\rho} \mathrm{W}$ is thus a Lie algebroid action and the invariant sections of $\mu^{\rho}$ form a Lie subalgebra which can be reduced to a quotient Lie algebroid structure on $\overline{\mu^{\rho}}: \overline{T^{\rho} \mathrm{W}}=T^{\rho} \mathrm{W} / G \rightarrow \overline{\mathrm{W}}=\mathrm{W} / G$. In this set-up, the reduced anchor map, defined as in $(8)$, is

$$
\overline{\rho^{\mu}}\left[\mathrm{v}, X_{\mathrm{w}}\right]=T \bar{\pi}^{\mathrm{W}}\left(\rho^{\mu}\left(\mathrm{v}, X_{\mathrm{w}}\right)\right)=T \bar{\pi}^{\mathrm{W}}\left(X_{\mathrm{w}}\right) .
$$


We will show now that we can in fact identify $\overline{\mu^{\rho}}: \overline{T^{\rho} \mathrm{W}} \rightarrow \overline{\mathrm{W}}$ with a prolongation bundle, namely the prolongation bundle $\bar{\mu}^{\bar{\rho}}: T^{\bar{\rho} \mathrm{W}} \rightarrow \overline{\mathrm{W}}$, formed by the quotient Lie algebroid $\bar{\tau}: \overline{\mathrm{V}} \rightarrow \bar{M}$ (with anchor map $\bar{\rho}$, as defined in (8) and bracket (7)) and the quotient subbundle $\bar{\mu}: \overline{\mathrm{W}} \rightarrow \bar{M}$.

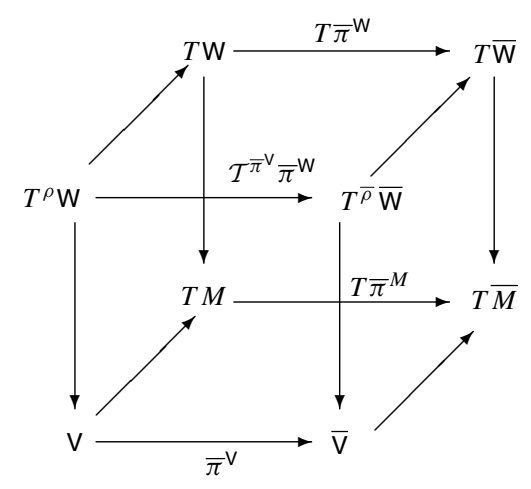

Proposition 3. The quotient prolongation Lie algebroid $\overline{\mu^{\rho}}: \overline{T^{\rho} \mathrm{W}} \rightarrow \overline{\mathrm{W}}$ and the prolongation Lie algebroid $\bar{\mu}^{\bar{\rho}}: T^{\bar{\rho} \overline{\mathrm{W}}} \rightarrow \overline{\mathrm{W}}$ are isomorphic as Lie algebroids. Moreover, $\operatorname{Sec}\left(\overline{\mu^{\rho}}\right), \operatorname{Sec}\left(\bar{\mu}^{\bar{\rho}}\right)$ and $\operatorname{Sec}^{I}\left(\mu^{\rho}\right)$ are isomorphic as Lie algebras.

Proof. Expression (12) for $\mathcal{T}^{\bar{\pi}^{\mathrm{v}}} \bar{\pi}^{\mathrm{W}}: T^{\rho} \mathrm{W} \rightarrow T^{\bar{\rho} \mathrm{W}}$ is

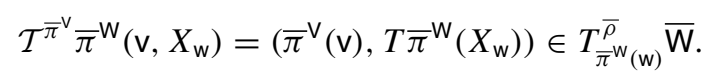

We will show that, for each $[\mathrm{w}] \in \overline{\mathrm{W}}$, the map

$$
\Sigma_{[\mathrm{w}]}:\left[\mathrm{v}, X_{\mathrm{w}}\right] \in{\overline{T^{\rho} \mathrm{W}_{[\mathrm{w}]}}} \mapsto \mathcal{T}_{\mathrm{w}}^{\bar{\pi}^{\mathrm{v}}} \bar{\pi}^{\mathrm{W}}\left(\mathrm{v}, X_{\mathrm{w}}\right) \in T_{[\mathrm{w}]}^{\bar{\rho}} \overline{\mathrm{W}}
$$

is a Lie algebroid isomorphism. First, we prove that the two spaces are isomorphic as vector spaces. From the considerations in section 2 about quotient bundles we know that, for each $\mathrm{w} \in \mathrm{W},\left(\bar{\pi}_{T^{\rho} \mathrm{W}}\right)_{\mathrm{w}}^{-1}:\left[\mathrm{v}, X_{\mathrm{w}}\right] \mapsto\left(\mathrm{v}, X_{\mathrm{w}}\right)$ is an isomorphism (it selects the unique representative of the class $\left[\mathrm{v}, X_{\mathrm{w}}\right]$ in the fibre at $\left.\mathrm{w}\right)$. Since $\Sigma_{[\mathrm{w}]}$ is in fact the composition $\mathcal{T}_{\mathrm{w}}^{\bar{\pi}^{\mathrm{v}}} \bar{\pi}^{\mathrm{W}} \circ\left(\bar{\pi}_{T^{\rho} \mathrm{W}}\right)_{\mathrm{w}}^{-1}$, we only need to show that, for each $\mathrm{w} \in \mathrm{W}$, the map $\mathcal{T}_{\mathrm{w}}^{\bar{\pi}^{\mathrm{v}}} \bar{\pi}^{\mathrm{W}}: T_{\mathrm{w}}^{\rho} \mathrm{W} \rightarrow T_{[\mathrm{w}]}^{\bar{\rho}} \overline{\mathrm{W}}$ is a linear isomorphism. The linearity is obvious. Since $\psi^{\mathrm{W}}$ and $\psi^{T \mathrm{~W}}=T \psi^{\mathrm{W}}$ are supposed to be vector bundle actions, $\mathrm{W}_{m} \simeq \overline{\mathrm{W}}_{[m]}$ and $T_{\mathrm{W}} \mathrm{W} \simeq T_{[\mathrm{w}]} \overline{\mathrm{W}}$. Therefore, clearly the dimensions of $T_{\mathrm{w}}^{\rho} \mathrm{W}$ and $T_{[\mathrm{w}]}^{\bar{\rho}} \overline{\mathrm{W}}$ will be the same. It remains to show that $\mathcal{T}_{\mathrm{w}}^{\bar{\pi}^{\mathrm{v}}} \bar{\pi}^{\mathrm{W}}$ is injective. Suppose that $\mathcal{T}_{\mathrm{w}}^{\bar{\pi}^{\mathrm{v}}} \bar{\pi}^{\mathrm{w}}\left(\mathrm{v}, X_{\mathrm{w}}\right)=0$, then, on the one hand, [v] $=0$, meaning that $\mathrm{v}=0$ and thus also $T \mu\left(X_{\mathrm{w}}\right)=0$. On the other hand, $T \bar{\pi}^{\mathrm{w}}\left(X_{\mathrm{w}}\right)=0$ which means that there exists a $\xi \in \mathfrak{g}=T_{e} G$ such that $X_{\mathrm{w}}=T_{e} \psi_{\mathrm{w}}^{\mathrm{W}}(\xi)\left(\psi_{\mathrm{w}}^{\mathrm{W}}: G \rightarrow W, g \mapsto g \mathrm{w}\right)$. As a result, $0=T \mu\left(X_{\mathrm{w}}\right)=T \mu\left(T_{e} \psi_{\mathrm{w}}^{\mathrm{W}}(\xi)\right)=T\left(\mu \circ \psi_{\mathrm{w}}^{\mathrm{W}}\right)(\xi)=T \psi_{m}^{M}(\xi)$ (where $\left.m=\mu(\mathrm{w})\right)$. Since $\psi_{m}^{M}$ is an injective immersion, it follows that $\xi=0$ and therefore also $X_{\mathrm{w}}=0$.

To prove now that $\Sigma$ is a Lie algebroid morphism (over the identity $\bar{W} \rightarrow \bar{W}$ ), we first check the admissibility condition $T$ id $\circ \bar{\rho}^{\mu}=\bar{\rho}^{\bar{\mu}} \circ \mathcal{T}^{\bar{\pi}^{\vee}} \bar{\pi}^{\mathrm{W}}$. When applied to an element [v, $X_{\mathrm{w}}$ ], the left-hand side gives $T \bar{\pi}^{\mathrm{W}}\left(X_{\mathrm{w}}\right)$ (by using (24)). This is in agreement with the righthand side, because also $\bar{\rho}^{\bar{\mu}}\left(\bar{\pi}^{\mathrm{v}}(\mathrm{v}), T \bar{\pi}^{\mathrm{W}}\left(X_{\mathrm{v}}\right)\right)=T \bar{\pi}^{\mathrm{W}}\left(X_{\mathrm{v}}\right)$. It remains to check the condition 
on the bracket, which, in this case, is equivalent to establishing a Lie algebra (iso)morphism $\Sigma_{*}$ between $\operatorname{Sec}\left(\overline{\mu^{\rho}}\right)$ and $\operatorname{Sec}\left(\bar{\mu}^{\bar{\rho}}\right)$.

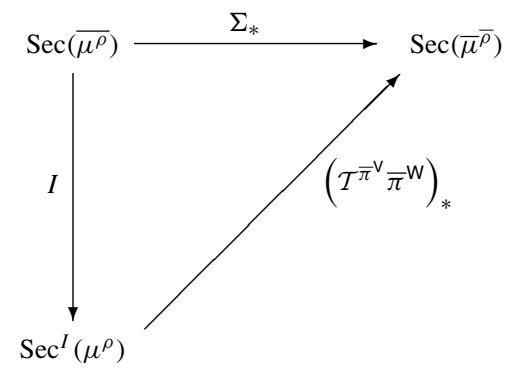

From our conclusions in section 2 , the projection $\bar{\pi}_{T^{\rho} \mathrm{W}}: T^{\rho} \mathrm{W} \rightarrow \overline{T^{\rho} \mathrm{W}}$ is a Lie algebroid morphism and it generates a Lie algebra isomorphism $(.)^{I}$ between $\operatorname{Sec}\left(\overline{\mu^{\rho}}\right)$ and the set of invariant sections $\operatorname{Sec}^{I}\left(\mu^{\rho}\right)$. Secondly, $\mathcal{T}^{\bar{\pi}^{\mathrm{v}}} \bar{\pi}^{\mathrm{W}}$ can be extended to a map $\left(\mathcal{T}^{\bar{\pi}^{\mathrm{N}}} \bar{\pi}^{\mathrm{W}}\right)_{*}: \operatorname{Sec}^{I}\left(\mu^{\rho}\right) \rightarrow \operatorname{Sec}\left(\bar{\mu}^{\bar{\rho}}\right)$ by means of

$$
\left(\mathcal{T}^{\bar{\pi}^{v}} \bar{\pi}^{\mathrm{W}}\right)_{*}(\mathcal{Z})([\mathrm{w}])=\mathcal{T}^{\bar{\pi}^{\mathrm{v}}} \bar{\pi}^{\mathrm{W}}\left(\mathcal{Z}\left(\left(\bar{\pi}_{m}^{\mathrm{W}}\right)^{-1}([\mathrm{w}])\right)\right) .
$$

It can easily be checked that, due to the supposed invariance of $\mathcal{Z}, m$ can be any element of $[m]=\bar{\tau}([\mathrm{w}])$ in this definition. $\left(\mathcal{T}^{\bar{\pi}^{\vee}} \bar{\pi}^{\mathrm{W}}\right)_{*}$ has an inverse $\left(\mathcal{T}^{\bar{\pi}^{\vee}} \bar{\pi}^{\mathrm{W}}\right)_{*}^{-1}: \operatorname{Sec}\left(\bar{\mu}^{\bar{\rho}}\right) \rightarrow \operatorname{Sec}^{I}\left(\mu^{\rho}\right)$, given by

$$
\left(\mathcal{T}^{\bar{\pi}^{\mathrm{v}}} \bar{\pi}^{\mathrm{w}}\right)_{*}^{-1}(\overline{\mathcal{Z}})(\mathrm{w})=\left(\mathcal{T}^{\bar{\pi}^{\mathrm{v}}} \bar{\pi}^{\mathrm{W}}\right)_{\mathrm{w}}^{-1}(\overline{\mathcal{Z}}([\mathrm{w}])),
$$

and is thus an isomorphism. Equation (25) in fact shows that $\left(\mathcal{T}^{\pi^{v}} \bar{\pi}^{\mathrm{w}}\right)_{*}(\mathcal{Z})$ is the unique section that is $\mathcal{T}^{\pi^{v}} \bar{\pi}^{\mathrm{W}}$-related to an invariant $\mathcal{Z}$. Moreover, since $\bar{\pi}^{\mathrm{V}}$ is a Lie algebroid morphism, also $\mathcal{T}^{\bar{\pi}^{\mathrm{v}}} \bar{\pi}^{\mathrm{W}}$ is one. The bracket $\left[\mathcal{Z}_{1}, \mathcal{Z}_{2}\right]$ is thus $\mathcal{T}^{\bar{\pi}^{\mathrm{v}}} \bar{\pi}^{\mathrm{W}}$-related to $\left[\left(\mathcal{T}^{\bar{\pi}^{\mathrm{v}}} \bar{\pi}^{\mathrm{W}}\right)_{*}\left(\mathcal{Z}_{1}\right),\left(\mathcal{T}^{\bar{\pi}^{\mathrm{v}}} \bar{\pi}^{\mathrm{W}}\right)_{*}\left(\mathcal{Z}_{2}\right)\right]$. Since also $\left(\mathcal{T}^{\bar{\pi}^{\mathrm{v}}} \bar{\pi}^{\mathrm{W}}\right)_{*}\left(\left[\mathcal{Z}_{1}, \mathcal{Z}_{2}\right]\right)$ is $\mathcal{T}^{\bar{\pi}^{\mathrm{v}}} \bar{\pi}^{\mathrm{W}}$-related to $\left[\mathcal{Z}_{1}, \mathcal{Z}_{2}\right]$, these two brackets must coincide, which proves that $\left(\mathcal{T}^{\mathrm{\pi}} \bar{\pi}^{\mathrm{W}}\right)_{*}$ is a Lie algebra isomorphism.

In conclusion, the composition of the maps $(.)^{I}$ and $\left(\mathcal{T}^{\pi^{\mathrm{v}}} \bar{\pi}^{\mathrm{W}}\right)_{*}$ is exactly the sought Lie algebra morphism $\Sigma_{*}: \operatorname{Sec}\left(\overline{\mu^{\rho}}\right) \rightarrow \operatorname{Sec}\left(\bar{\mu}^{\bar{\rho}}\right)$ we were looking for.

The observation that $\mathcal{T}^{\bar{\pi}^{v}} \bar{\pi}^{\mathrm{W}}$ is a Lie algebroid morphism means that, for the exterior derivatives $\tilde{\mathrm{d}}$ on $\bigwedge\left(\mu^{\rho}\right)$ and $\overline{\tilde{\mathrm{d}}}$ on $\bigwedge\left(\bar{\mu}^{\bar{\rho}}\right)$,

$$
\tilde{\mathrm{d}} \circ\left(\mathcal{T}^{\bar{\pi}^{\vee}} \bar{\pi}^{\mathrm{W}}\right)^{*}=\left(\mathcal{T}^{\bar{\pi}^{\vee}} \bar{\pi}^{\mathrm{W}}\right)^{*} \circ \overline{\tilde{\mathrm{d}}},
$$

where $\left(\mathcal{T}^{\bar{\pi}^{\vee}} \bar{\pi}^{\mathrm{W}}\right)^{*}: \bigwedge^{k}\left(\bar{\mu}^{\bar{\rho}}\right) \rightarrow \bigwedge^{k}\left(\mu^{\rho}\right)$ is defined as in (4). We can, of course prove a similar result for $\mathcal{T}^{\bar{\pi}^{v}} \bar{\pi}^{\mathrm{V}}$.

Corollary 1. If $\mathrm{d}$ is the exterior derivative on $\bigwedge\left(\tau^{\rho}\right)$ and $\overline{\mathrm{d}}$ on $\bigwedge\left(\bar{\tau}^{\bar{\rho}}\right)$, then $\mathcal{T}^{\bar{\pi}^{\vee}} \bar{\pi}^{\vee}$ is a Lie algebroid morphism over $\bar{\pi}^{\mathrm{V}}$, i.e.,

$$
\mathrm{d} \circ\left(\mathcal{T}^{\bar{\pi}^{\vee}} \bar{\pi}^{\vee}\right)^{*}=\left(\mathcal{T}^{\bar{\pi}^{\vee}} \bar{\pi}^{\vee}\right)^{*} \circ \overline{\mathrm{d}} .
$$

The quotient prolongation Lie algebroid $\overline{\tau^{\rho}}: \overline{T^{\rho} \mathrm{V}} \rightarrow \overline{\mathrm{V}}$ and the prolongation Lie algebroid $\bar{\tau}^{\bar{\rho}}: T^{\bar{\rho} \bar{V}} \rightarrow \overline{\mathrm{V}}$ are isomorphic as Lie algebroids. Moreover, $\operatorname{Sec}\left(\overline{\tau^{\rho}}\right), \operatorname{Sec}\left(\bar{\tau}^{\bar{\rho}}\right)$ and $\operatorname{Sec}^{I}\left(\tau^{\rho}\right)$ are isomorphic as Lie algebras.

Much of what has just been said also applies to the prolongation bundle $\mu^{\lambda}: T^{\lambda} \mathrm{W} \rightarrow \mathrm{W}$ even though it does not carry a Lie algebroid structure. The maps $\bar{i}$ and $\bar{\rho}$ induce a map 
$\bar{\lambda}=\bar{\rho} \circ \bar{i}: \overline{\mathrm{W}} \rightarrow T \bar{M}$. The quotient projection $\bar{\pi}^{\mathrm{W}}$ is admissible for the 'anchors' $\lambda$ and $\bar{\lambda}$, in the sense that $T \bar{\pi}^{M} \circ \lambda=\bar{\lambda} \circ \bar{\pi}^{\mathrm{W}}$ (because of (8) and (23)). Therefore, it is easy to see that the map $\mathcal{T}^{\bar{\pi}^{\mathrm{W}}} \bar{\pi}^{\mathrm{W}}: T^{\lambda} \mathrm{W} \rightarrow T^{\bar{\lambda}} \overline{\mathrm{W}}$ is still well defined and admissible with respect to $\lambda^{\mu}$ and $\bar{\lambda}^{\bar{\mu}}$ :

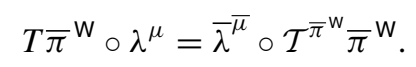

Lemma 2. The diagram

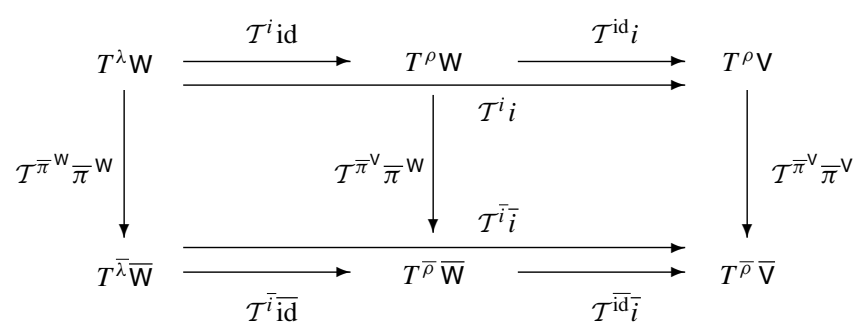

is completely commutative.

Proof. The map id stands for both the identity maps on $\overline{\mathrm{V}}$ and $\overline{\mathrm{W}}$. $\mathcal{T}^{\overline{\mathrm{i}}} \bar{i}$ is defined, for the quotient bundles, as in (16). The proof is obvious.

In the case of the prolongations $\mu^{\lambda}$ and $\bar{\mu}^{\bar{\lambda}}$, the operators $\delta: \bigwedge\left(\mu^{\rho}\right) \rightarrow \bigwedge\left(\mu^{\lambda}\right)$, $\bar{\delta}: \bigwedge\left(\bar{\mu}^{\bar{\rho}}\right) \rightarrow \bigwedge\left(\bar{\mu}^{\bar{\lambda}}\right), \Delta: \bigwedge\left(\tau^{\rho}\right) \rightarrow \bigwedge\left(\mu^{\lambda}\right)$ and $\bar{\Delta}: \bigwedge\left(\bar{\tau}^{\bar{\rho}}\right) \rightarrow \bigwedge\left(\bar{\mu}^{\bar{\lambda}}\right)$ (defined in (18) and (20)) have similar properties as the exterior derivatives.

Corollary 2. The bundles $\overline{\mu^{\lambda}}: \overline{T^{\lambda} \mathrm{W}} \rightarrow \overline{\mathrm{W}}$ and $\bar{\mu}^{\bar{\lambda}}: T^{\bar{\lambda}} \overline{\mathrm{W}} \rightarrow \overline{\mathrm{W}}$ are isomorphic as vector bundles. Moreover,

$\delta \circ\left(\mathcal{T}^{\bar{\pi}^{\mathrm{v}}} \bar{\pi}^{\mathrm{W}}\right)^{*}=\left(\mathcal{T}^{\bar{\pi}^{\mathrm{w}}} \bar{\pi}^{\mathrm{W}}\right)^{*} \circ \bar{\delta} \quad$ and $\quad \Delta \circ\left(\mathcal{T}^{\bar{\pi}^{\mathrm{v}}} \bar{\pi}^{\mathrm{V}}\right)^{*}=\left(\mathcal{T}^{\bar{\pi}^{\mathrm{w}}} \bar{\pi}^{\mathrm{w}}\right)^{*} \circ \bar{\Delta}$.

Proof. The first statement can easily be deduced from the proof of proposition 3. The identity $\mathcal{T}^{\bar{i}} \overline{\overline{\mathrm{i}}} \circ \mathcal{T}^{\bar{\pi}^{\mathrm{w}}} \bar{\pi}^{\mathrm{w}}=\mathcal{T}^{\bar{\pi}^{\mathrm{v}}} \bar{\pi}^{\mathrm{w}} \circ \mathcal{T}^{i} i d$, leads to the identity $\left(\mathcal{T}^{\bar{\pi}^{\mathrm{w}}} \bar{\pi}^{\mathrm{w}}\right)^{*} \circ\left(\mathcal{T}^{\bar{i}} \overline{\overline{\mathrm{i}}}\right)^{*}=$ $\left(\mathcal{T}^{i} \text { id }\right)^{*} \circ\left(\mathcal{T}^{\bar{\pi}^{v}} \bar{\pi}^{\mathrm{W}}\right)^{*}$ on forms. Then, from property (27) we can deduce that $\delta \circ\left(\mathcal{T}^{\bar{\pi}^{v}} \bar{\pi}^{\mathrm{W}}\right)^{*}=$ $\left(\mathcal{T}^{i} \mathrm{id}\right)^{*} \circ \tilde{\mathrm{d}} \circ\left(\mathcal{T}^{\bar{\pi}^{\mathrm{v}}} \bar{\pi}^{\mathrm{W}}\right)^{*}=\left(\mathcal{T}^{i} \mathrm{id}\right)^{*} \circ\left(\mathcal{T}^{\bar{\pi}^{\mathrm{v}}} \bar{\pi}^{\mathrm{W}}\right)^{*} \circ \overline{\tilde{\mathrm{d}}}=\left(\mathcal{T}^{\bar{\pi}^{\mathrm{w}}} \bar{\pi}^{\mathrm{W}}\right)^{*} \circ\left(\mathcal{T}^{\bar{i}} \overline{\mathrm{id}}\right)^{*} \circ \overline{\mathrm{d}}=\left(\mathcal{T}^{\bar{\pi}^{\mathrm{w}}} \bar{\pi}^{\mathrm{w}}\right)^{*} \circ \bar{\delta}$. The last property follows in a similar way from the identity $\mathcal{T}^{\bar{i}} \bar{i} \circ \mathcal{T}^{\bar{\pi}^{\mathrm{w}}} \bar{\pi}^{\mathrm{W}}=\mathcal{T}^{\bar{\pi}^{\mathrm{v}}} \bar{\pi}^{\mathrm{v}} \circ \mathcal{T}^{i} i$ and property (28).

\section{Reduction of Lagrangian systems on a subbundle of a Lie algebroid}

Let us come back to the Lagrangian systems (21) on a subbundle of a Lie algebroid.

Definition 5. A Lagrangian $L \in C^{\infty}(\mathrm{V})$ is said to be reducible if it is invariant under the action of the Lie group, i.e. $L(g \mathrm{v})=L(\mathrm{v})$.

The reduced Lagrangian is then the function $\bar{L} \in C^{\infty}(\overline{\mathrm{V}})$, satisfying $L=\bar{L} \circ \bar{\pi}^{\vee}$, or $\bar{L}([\mathrm{v}])=L(\mathrm{v})$. This function has of course its own 1-form $\theta_{\bar{L}} \in \bigwedge^{1}\left(\bar{\tau}^{\bar{\rho}}\right)$ and energy function $E_{\bar{L}} \in C^{\infty}(\overline{\mathrm{V}})$. We will show now that equation (21) for $L$ projects on the Lagrangian equation for $\bar{L}$ (on the subbundle $\bar{\mu}$ of the quotient Lie algebroid $\bar{\tau}$ ), given by

$$
i_{\bar{\Gamma}} \bar{\Delta} \theta_{\bar{L}}=-\bar{\Delta} E_{\bar{L}}
$$


Lemma 3. The vertical endomorphisms $S^{\tau}: T^{\rho} \mathrm{V} \rightarrow T^{\rho} \mathrm{V}$ and $S^{\bar{\tau}}: T^{\bar{\rho} \bar{V}} \rightarrow T^{\bar{\rho} \mathrm{V}}$ satisfy the relation

$$
S^{\bar{\tau}} \circ \mathcal{T}^{\bar{\pi}^{\vee}} \bar{\pi}^{\vee}=\mathcal{T}^{\bar{\pi}^{\vee}} \bar{\pi}^{\vee} \circ S^{\tau} .
$$

On functions $\bar{f} \in C^{\infty}(\bar{M})$, the action of the Liouville sections is related by

$$
\rho^{\tau}\left(\mathcal{C}^{\tau}\right)\left(\bar{f} \circ \bar{\pi}^{\vee}\right)=\left(\bar{\rho}^{\bar{\tau}}\left(\mathcal{C}^{\bar{\tau}}\right) \bar{f}\right) \circ \bar{\pi}^{\mathrm{V}} .
$$

Proof. By definition of $S^{\bar{\tau}}$, we have $S^{\bar{\tau}}\left(\mathcal{T}^{\bar{\pi}^{\mathrm{v}}} \bar{\pi}^{\mathrm{v}}\left(\mathrm{v}, X_{\mathrm{a}}\right)\right)=\left(\bar{j}\left(\bar{\pi}^{\mathrm{v}}(\mathrm{v}), T \bar{\pi}^{\mathrm{v}}\left(X_{\mathrm{a}}\right)\right)\right)^{\bar{V}}=$ $\left(\bar{\pi}^{\vee}(\mathrm{a}), \bar{\pi}^{\mathrm{V}}(\mathrm{v})\right)^{\bar{V}}=(\overline{0}, \bar{Y})$, with

$$
\bar{Y}(\bar{f})=\left.\frac{\mathrm{d}}{\mathrm{d} t} \bar{f}\left(\bar{\pi}^{\mathrm{v}}(\mathrm{a})+t \bar{\pi}^{\mathrm{v}}(\mathrm{v})\right)\right|_{t=0}, \quad \forall \bar{f} \in C^{\infty}(\bar{M}) .
$$

On the other hand, $\mathcal{T}^{\bar{\pi}^{\vee}} \bar{\pi}^{\mathrm{v}}\left(S^{\tau}\left(\mathrm{v}, X_{\mathrm{a}}\right)\right)=\mathcal{T}^{\bar{\pi}^{\vee}} \bar{\pi}^{\mathrm{v}}\left((\mathrm{a}, \mathrm{v})^{V}\right)=\mathcal{T}^{\bar{\pi}^{\vee}} \bar{\pi} \mathrm{\vee}(0, Y)=\left(\overline{0}, T \bar{\pi}^{\mathrm{v}}(Y)\right)$, where

$$
Y(f)=\left.\frac{\mathrm{d}}{\mathrm{d} t} f(\mathrm{a}+t \mathrm{v})\right|_{t=0}, \quad \forall f \in C^{\infty}(M),
$$

and thus $T \bar{\pi}^{\mathrm{v}}(Y)(\bar{f})=Y\left(\bar{f} \circ \bar{\pi}^{\mathrm{v}}\right)=\left.\frac{\mathrm{d}}{\mathrm{d} t} f\left(\bar{\pi}^{\mathrm{v}}(\mathrm{a}+t \mathrm{v})\right)\right|_{t=0}$, from which we can conclude that $T \bar{\pi}^{\vee}(Y)=\bar{Y}$ indeed.

To prove the second property, if $\rho^{\tau}\left(\mathcal{C}^{\tau}\right)(\mathrm{v})=X$, then $\rho^{\tau}\left(\mathcal{C}^{\tau}\right)\left(\bar{f} \circ \bar{\pi}^{\mathrm{v}}\right)(\mathrm{v})=X\left(\bar{f} \circ \bar{\pi}^{\mathrm{v}}\right)=$ $\left.\frac{\mathrm{d}}{\mathrm{d} t} f\left(\bar{\pi}^{\mathrm{V}}(\mathrm{v}+t \mathrm{v})\right)\right|_{t=0}$. This is, again, exactly $\left(\bar{\rho}^{\bar{\tau}}\left(\mathcal{C}^{\bar{\tau}}\right) \bar{f}\right)\left(\bar{\pi}^{\mathrm{V}}(\mathrm{v})\right)=\left(\bar{\rho}^{\bar{\tau}}\left(\mathcal{C}^{\bar{\tau}}\right)\left(\bar{\pi}^{\mathrm{v}}(\mathrm{v})\right)\right)(\bar{f})=$ $\left.\frac{\mathrm{d}}{\mathrm{d} t} \bar{f}\left(\bar{\pi}^{\mathrm{v}}(\mathrm{v})+t \bar{\pi}^{\mathrm{v}}(\mathrm{v})\right)\right|_{t=0}$.

Lemma 4. The Poincaré-Cartan forms and the energy functions of $L$ and $\bar{L}$ are related in such a way that $\left(\mathcal{T}^{\bar{\pi}^{\vee}} \bar{\pi}^{\vee}\right)^{*} \theta_{\bar{L}}=\theta_{L}$ and $\left(\mathcal{T}^{\bar{\pi}^{\vee}} \bar{\pi}^{\vee}\right)^{*} E_{\bar{L}}=E_{L}$.

Proof. From $L=\bar{L} \circ \bar{\pi}^{\vee}$ (or $\left(\mathcal{T}^{\bar{\pi}^{\vee}} \bar{\pi}^{\vee}\right)^{*} \bar{L}=L$ ) and property (28) it follows that $\mathrm{d} L=\left(\mathcal{T}^{\bar{\pi}^{\vee}} \bar{\pi} \vee\right)^{*} \overline{\mathrm{d} L}$. Property (29) is equivalent to $S^{\tau} \circ\left(\mathcal{T}^{\bar{\pi}^{\vee}} \bar{\pi} \vee\right)^{*}=\left(\mathcal{T}^{\bar{\pi}^{\vee}} \bar{\pi}^{\vee}\right)^{*} \circ S^{\bar{\tau}}$ for the action on 1-forms. Together with the previous observation, it follows that $\theta_{L}=\left(\mathcal{T}^{\bar{\pi}^{\vee}} \bar{\pi}^{\vee}\right)^{*} \theta_{\bar{L}}$. Similarly, another way of writing (30) is $\rho^{\tau}\left(\mathcal{C}^{\tau}\right) \circ\left(\mathcal{T}^{\bar{\pi}^{\vee}} \bar{\pi}^{\vee}\right)^{*}=\left(\mathcal{T}^{\bar{\pi}^{\vee}} \bar{\pi}^{\vee}\right)^{*} \circ \bar{\rho}^{\bar{\tau}}\left(\mathcal{C}^{\bar{\tau}}\right)$ from which it follows that $E_{L}=\left(\mathcal{T}^{\bar{\pi}^{\vee}} \bar{\pi}^{\mathrm{W}}\right)^{*} E_{\bar{L}}$ or $E_{L}=E_{\bar{L}} \circ \bar{\pi}^{\mathrm{V}}$.

Theorem 1. Suppose $L$ is a reducible regular Lagrangian on $\mathrm{V}$. Then, also $\bar{L}$ is regular. Moreover the Lagrangian section $\Gamma \in \operatorname{Sec}\left(\mu^{\lambda}\right)$ is invariant and the solutions of the nonholonomic equations on $L$ (i.e. the integral curves of $\left.\lambda^{\mu}(\Gamma)\right)$ project to those for the reduced Lagrangian $\bar{L}$ (i.e. the integral curves of $\bar{\lambda}^{\bar{\mu}}(\bar{\Gamma})$ ).

Proof. From the previous lemma it is clear that

$$
\Delta \theta_{L}=\Delta \circ\left(\mathcal{T}^{\bar{\pi}^{\mathrm{v}} \bar{\pi}}\right)^{*} \theta_{\bar{L}}=\left(\mathcal{T}^{\bar{\pi}^{\mathrm{w}} \bar{\pi}} \mathrm{W}\right)^{*} \circ \bar{\Delta} \theta_{\bar{L}} .
$$

Since each $\mathcal{T}_{\mathrm{w}}^{\bar{\pi}^{\mathrm{w}} \bar{\pi}} \mathrm{w}$ is an isomorphism, this relation ensures that $\bar{\Delta} \theta_{\bar{L}}$ will also be nondegenerate. Further, also

$$
\Delta E_{L}=\left(\Delta \circ\left(\mathcal{T}^{\bar{\pi}^{\vee}} \bar{\pi}^{\vee}\right)^{*}\right) E_{\bar{L}}=\left(\left(\mathcal{T}^{\bar{\pi}^{\mathrm{w}}} \bar{\pi}^{\mathrm{w}}\right)^{*} \circ \bar{\Delta}\right) E_{\bar{L}},
$$

from which it follows that

$$
\mathcal{T}_{\mathrm{w}}^{\bar{\pi}^{\mathrm{w}}} \bar{\pi}^{\mathrm{w}}(\Gamma(\mathrm{w}))=\bar{\Gamma}\left(\bar{\pi}^{\mathrm{w}}(\mathrm{w})\right) .
$$


It is further obvious that also $T \bar{\pi}^{\mathrm{w}}\left(\lambda^{\mu}(\Gamma(\mathrm{w}))\right)=\bar{\lambda}^{\bar{\mu}}\left(\bar{\Gamma}\left(\bar{\pi}^{\mathrm{w}}(\mathrm{w})\right)\right)$ and therefore $\lambda^{\mu}(\Gamma)$ and $\bar{\lambda}^{\bar{\mu}}(\bar{\Gamma})$ must be $\bar{\pi}^{\mathrm{W}}$-related. Observe finally that (31) indicates that both $\Gamma(\mathrm{w})$ and $\Gamma(\mathrm{gw})$ project to the same equivalence class, which can only mean that $\Gamma(g \mathrm{w})=g \Gamma(\mathrm{w})$, or $\Gamma \in \operatorname{Sec}^{I}\left(\mu^{\lambda}\right)$. With a similar definition as (25), we can thus write that $\bar{\Gamma}=\left(\mathcal{T}^{\bar{\pi}^{\mathrm{w}}} \bar{\pi}^{\mathrm{W}}\right)_{*}(\Gamma)$.

\section{Lagrangian reduction by stages}

In this section, we prove that the category of Lagrangians systems on a subbundle of a Lie algebroid is stable under successive reduction. We start by recalling some known facts which can be found in [2]. Suppose that $\bar{\pi}^{M}: M \rightarrow \bar{M}=M / G$ is a principal fibre bundle and that $N \subset G$ is a normal subgroup of $G$. Since the action $\psi^{M}$ of $G$ on $M$ can be restricted to $N$, it makes perfect sense to speak of the quotient $\hat{M}=M / N$. In fact, $M$ is also a principal fibre bundle with respect to this restricted action, $\hat{\pi}^{M}: M \rightarrow \hat{M}$. Moreover, we can define an action of $H=G / N$ on $\hat{M}$ by means of

$$
[\mathrm{g}]_{N}[\mathrm{~m}]_{N}=[\mathrm{gm}]_{N},
$$

which gives $\hat{M}$ the structure of a principal fibre bundle with structure group $H, \hat{\pi}^{M}: \hat{M} \rightarrow$ $\hat{\hat{M}}=\hat{M} / H$. Another way of writing (32) is then

$$
\psi_{[g]_{N}}^{\hat{M}} \circ \hat{\pi}^{M}=\hat{\pi}^{M} \circ \psi_{g}^{M} .
$$

Further, the map

$$
\beta^{M}: \bar{M} \rightarrow \hat{\hat{M}}, \quad[m]_{G} \rightarrow\left[[m]_{N}\right]_{H}
$$

is a diffeomorphism.

Suppose now that $\tau: \mathrm{V} \rightarrow M$ is a vector bundle, equipped with a $G$-vector bundle action $\psi^{\mathrm{V}}$. Again, $\psi^{\vee}$ can be restricted to an $N$-vector bundle action on $\tau$. Then, both $\bar{\tau}: \overline{\mathrm{V}} \rightarrow \bar{M}$ and $\hat{\tau}: \hat{\mathrm{V}}=\mathrm{V} / N \rightarrow \hat{M}$ are vector bundles. We will denote the submersion $\mathrm{V} \rightarrow \hat{\mathrm{V}}$ by $\hat{\pi}^{\mathrm{V}}$. There is also an induced vector bundle action of $H$ on $\hat{\tau}$, given by $\psi_{[g]_{N}}^{\hat{\mathrm{V}}}: \hat{\mathrm{V}} \rightarrow \hat{\mathrm{V}}:[\mathrm{v}]_{N} \mapsto[g]_{N}[\mathrm{v}]_{N}=[g \mathrm{v}]_{N}$, or

$$
\psi_{[g]_{N}}^{\hat{\mathrm{V}}} \circ \hat{\pi}^{\mathrm{V}}=\hat{\pi}^{\mathrm{V}} \circ \psi_{g}^{\mathrm{V}} .
$$

Therefore, also the quotient $\hat{\hat{\tau}}: \hat{\hat{V}}=\hat{\mathrm{V}} / H \rightarrow \hat{\hat{M}}$ is a vector bundle. Again, there exists a well-defined (vector bundle) isomorphism

$$
\beta^{\mathrm{v}}: \overline{\mathrm{V}} \rightarrow \hat{\mathrm{V}}, \quad[\mathrm{v}]_{G} \rightarrow\left[[\mathrm{v}]_{N}\right]_{H}
$$

over $\beta^{M}$. In other words,

$$
\beta_{[m]_{G}}^{\mathrm{V}} \circ \bar{\pi}_{m}^{\mathrm{V}}=\hat{\hat{\pi}}_{[m]_{N}}^{\mathrm{V}} \circ \hat{\pi}_{m}^{\mathrm{V}}
$$

where $\hat{\hat{\pi}}^{\mathrm{v}}$ is the projection $\hat{\mathrm{V}} \rightarrow \hat{\hat{\mathrm{V}}} ;[\mathrm{v}]_{N} \mapsto\left[[\mathrm{v}]_{N}\right]_{H}\left(\right.$ over $\left.\hat{\hat{\pi}}^{M}: \hat{M} \rightarrow \hat{\hat{M}} ;[m]_{N} \mapsto\left[[m]_{N}\right]_{H}\right)$.

Next, let $L$ be a (regular) Lagrangian on the Lie algebroid $\tau: \mathrm{V} \rightarrow M$ and $\mu: \mathrm{W} \rightarrow M$ a subbundle of $\tau$. The three conditions, necessary to perform an reduction by means of $G$ are as follows: (i) $L$ is a $G$-invariant Lagrangian; (ii) all $\psi_{g}^{\vee}$ are Lie algebroid isomorphisms; (iii) the $G$-action $\psi^{\vee}$ restricts to W. To explain how successive reduction works, we will from now on assume that these conditions are satisfied and we will verify that the induced actions of $N$ and $H$ satisfy similar conditions.

The first stage of the reduction is the reduction by the Lie group $N$. Since the Lagrangian is also obviously invariant under the $N$-action, it gives rise to a Lagrangian $\hat{L} \in C^{\infty}(\hat{\mathrm{V}})$ defined by $\hat{L}\left([\mathrm{v}]_{N}\right)=L(\mathrm{v})$. We will use, e.g., the notation $\operatorname{Sec}^{I, G}(\tau)$ for the set of the 
$\tau$-sections that are invariant under the action of $G$. Although $\operatorname{Sec}^{I, G}(\tau) \subset \operatorname{Sec}^{I, N}(\tau)$, also $\operatorname{Sec}^{I, N}(\tau)$ is a Lie subalgebra of $\operatorname{Sec}(\tau)$, because all $\psi_{n}^{\vee}: \mathrm{V} \rightarrow \mathrm{V}(n \in N)$ are Lie algebroid isomorphisms. Therefore, $\hat{\tau}$ can be given the structure of a Lie algebroid with a bracket and anchor $\hat{\rho}: \hat{V} \rightarrow T \hat{M}$ defined as in (7) and (8). From the third assumption, it follows that the restriction of $\psi^{\mathrm{v}}$ to $N$ restricts in turn to an action of $N$ on $\mathrm{W}$, so we can define the quotient vector bundle $\hat{\mu}: \hat{\mathrm{W}} \rightarrow \hat{M}$. Therefore, all conditions to perform a reduction by $N$ are satisfied and the Lagrangian equations for $L \in C^{\infty}(\mathrm{V})$ on the subbundle $\mu$ of $\tau$ will project to reduced equations for $\hat{L} \in C^{\infty}(\hat{M})$ on the subbundle $\hat{\mu}$ of the quotient Lie algebroid $\hat{\tau}: \hat{V} \rightarrow \hat{M}$.

The second reduction is the one by $H=G / N$. We can easily verify that the Lagrangian $\hat{L}$ is invariant under the $H$-action. Indeed,

$$
\hat{L}\left([g]_{N}[\mathrm{v}]_{N}\right)=\hat{L}\left([g \mathrm{v}]_{N}\right)=L(g \mathrm{v})=L(\mathrm{v})=\hat{L}\left([\mathrm{v}]_{N}\right) .
$$

Therefore, it is possible to reduce $\hat{L}$ to a Lagrangian $\hat{\hat{L}} \in C^{\infty}(\hat{\hat{V}}=\hat{V} / H)$. Also, the condition on the constrained action is satisfied. Indeed, since $\hat{\mathrm{W}} \rightarrow \hat{M}$ is a subbundle of $\hat{\mathrm{V}} \rightarrow \hat{M}$, the reduced action of $H$ on $\hat{V}$ restricts exactly to the reduced action of $H$ on $\hat{W}$. We can therefore introduce the vector bundle $\hat{\hat{W}}=\hat{\mathrm{W}} / H \rightarrow \hat{\hat{M}}=\hat{M} / H$ and our programme further will consist in showing that each $\psi_{[g]_{N}}^{\hat{V}}$ is a Lie algebroid morphism. We remark first that the following diagram is commutative.

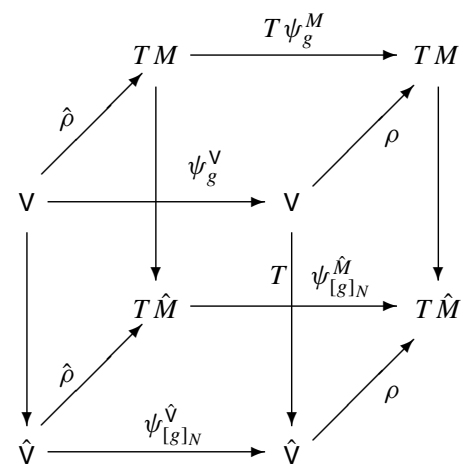

Proposition 4. For each $[g]_{N}, \psi_{[g]_{N}}^{\hat{\mathrm{V}}}: \hat{\mathrm{V}} \rightarrow \hat{\mathrm{V}}$ is a Lie algebroid isomorphism (over $\psi_{[g]_{N}}^{\hat{M}}$ ).

Proof. The admissibility $T \psi_{[g]_{N}}^{\hat{M}} \circ \hat{\rho}=\hat{\rho} \circ \psi_{[g]_{N}}^{\hat{V}}$ is clear from the diagram. Indeed, when applied to $[\mathrm{v}]_{N}$ (and taking into account the definition (8) for $\hat{\rho}$ ), the left-hand side is $T\left(\psi_{[g]_{N}}^{\hat{M}} \circ \hat{\pi}^{M}\right)(\rho(\mathrm{v}))$. In view of (33), this equals the right-hand side $T\left(\hat{\pi}^{M} \circ \psi_{g}^{\hat{M}}\right)(\rho(\mathrm{v}))$.

Further, with every section $\hat{s}$ in $\hat{\tau}$, we can identify a $N$-invariant section $\hat{s}^{I, N}$ of $\tau$ (see (1)), by means of

$$
\hat{\mathbf{s}}^{I, N}(m)=\left(\hat{\pi}_{m}^{\vee}\right)^{-1}\left(\hat{\mathbf{s}}\left([m]_{N}\right)\right) .
$$

Now, if $\hat{\mathrm{s}}_{1}$ is $\psi_{[g]_{N}}^{\hat{\mathrm{V}}}$-related to $\hat{\mathrm{s}}_{2}$ (for a specific $[g]_{N}$ ), then $\hat{\mathrm{s}}_{1}^{I, N}$ will be $\psi_{g}^{\mathrm{V}}$-related to $\hat{\mathrm{s}}_{2}^{I, N}$ (for any $\left.g \in[g]_{N}\right)$. Indeed, we have

$$
\begin{aligned}
\hat{\mathrm{s}}_{1}^{I, N}(g m) & =\left(\hat{\pi}_{g m}^{\mathrm{V}}\right)^{-1}\left(\hat{\mathrm{s}}_{1}\left([g]_{N}[m]_{N}\right)\right)=\left(\hat{\pi}_{g m}^{\mathrm{V}}\right)^{-1}\left([g]_{N} \hat{\mathrm{s}}_{1}\left([m]_{N}\right)\right) \\
& =g\left(\hat{\pi}_{m}^{\mathrm{V}}\right)^{-1}\left(\hat{\mathrm{s}}_{2}\left([m]_{N}\right)\right)=g \hat{\mathbf{s}}_{2}^{I, N}(m),
\end{aligned}
$$

where we have used the fact that $\hat{\pi}_{g m}^{\mathrm{V}}=\psi_{[g]_{N}}^{\hat{\mathrm{V}}} \circ \hat{\pi}_{m}^{\mathrm{V}} \circ \psi_{g-1}^{\mathrm{V}}$ and therefore $\left(\hat{\pi}_{g m}^{\mathrm{V}}\right)^{-1}=$ $\psi_{g}^{\mathrm{V}} \circ\left(\hat{\pi}_{m}^{\mathrm{V}}\right)^{-1} \circ \psi_{[g]_{N}^{-1}}^{\hat{\mathrm{V}}}$. Also the converse is true: if two $N$-invariant sections $\hat{\mathrm{s}}_{1}^{I, N}$ and $\hat{\mathrm{s}}_{2}^{I, N}$ of $\tau$ are $\psi_{g}^{\mathrm{V}}$-related, then $\hat{\mathrm{s}}_{1}$ and $\hat{\mathrm{s}}_{2}$ are $\psi_{[g]_{N}}^{\hat{\mathrm{v}}}$-related. 
Suppose now that also $\hat{r}_{1}$ is $\psi_{[g]_{N}}^{\hat{v}}$-related to a certain $\hat{r}_{2}$. Then, by definition (7), $\left[\hat{\mathrm{r}}_{1}, \hat{\mathrm{s}}_{1}\right]^{I, N}=\left[\hat{\mathrm{r}}_{1}^{I, N}, \hat{\mathrm{s}}_{1}^{I, N}\right]$. Moreover, since by assumption each $\psi_{g}^{\mathrm{V}}$ is a Lie algebroid isomorphism this last bracket is $\psi_{g}^{\mathrm{V}}$-related to the $N$-invariant bracket $\left[\hat{\mathrm{r}}_{2}^{I, N}, \hat{\mathrm{s}}_{2}^{I, N}\right]=\left[\hat{\mathrm{r}}_{2}, \hat{\mathrm{s}}_{2}\right]^{I, N}$. It follows that $\left[\hat{r}_{1}, \hat{s}_{1}\right]$ and $\left[\hat{r}_{2}, \hat{s}_{2}\right]$ are $\psi_{[g]_{N}}^{\hat{V}}$-related and thus we may conclude, that for each $[g]_{N}, \psi_{[g]_{N}}^{\hat{V}}$ is a Lie algebroid isomorphism.

The reduction process can now be continued: the quotient Lie algebroid $\hat{\tau}: \hat{\mathrm{V}} \rightarrow \hat{M}$ induces a quotient Lie algebroid structure on $\hat{\hat{\tau}}: \hat{\hat{V}} \rightarrow \hat{\hat{M}}$ (with the anchor $\hat{\hat{\rho}}: \hat{\hat{V}} \rightarrow \hat{\hat{M}}$ ). Equations (19) for the Lagrangian $L$ on the subbundle $\mu$ of the Lie algebroid $\tau$, reduce therefore, in two stages (first $N$, then $H$ ), to similar equations for the Lagrangian $\hat{\hat{L}}$ on the subbundle $\hat{\hat{\mu}}$ of the Lie algebroid $\hat{\hat{\tau}}$. We will show that this reduction is equivalent to the direct reduction under the action of $G$. for $\beta^{\mathrm{W}}$,

Recall first that $\beta^{\vee}$ (see (36)) is a (vector bundle) isomorphism. With a similar definition

$$
\beta_{[m]_{G}}^{\mathrm{W}} \circ \bar{\pi}_{m}^{\mathrm{W}}=\hat{\hat{\pi}}_{[m]_{N}}^{\mathrm{W}} \circ \hat{\pi}_{m}^{\mathrm{W}}
$$

also $\bar{\mu}$ and $\hat{\hat{\mu}}$ are isomorphic as vector bundles. Moreover, if $\bar{L} \in C^{\infty}(\overline{\mathrm{V}})$ is the Lagrangian, obtained after a reduction by the action of $G$, then

$$
\bar{L}\left([\mathrm{v}]_{G}\right)=\hat{\hat{L}}\left(\beta_{\mathrm{v}}\left([\mathrm{v}]_{G}\right)\right)=L(\mathrm{v}) .
$$

We will now show that the Lie algebroid structures on $\bar{\tau}$ and $\hat{\hat{\tau}}$ are also isomorphic.

Proposition 5. $\beta^{\vee}$ is a Lie algebroid isomorphism $\left(\right.$ over $\left.\beta^{M}\right) \cdot \operatorname{Sec}^{I, G}(\tau), \operatorname{Sec}(\bar{\tau}), \operatorname{Sec}^{I, H}(\hat{\tau})$ and $\operatorname{Sec}(\hat{\hat{\tau}})$ are all isomorphic as Lie algebras.

Proof. We begin by checking the admissibility property, or $T \beta^{M} \circ \bar{\rho}\left([\mathrm{v}]_{G}\right)=\hat{\hat{\rho}} \circ \beta^{\mathrm{V}}\left([\mathrm{v}]_{G}\right)$. The left side is $T\left(\beta^{M} \circ \bar{\pi}^{M}\right)(\rho(\mathrm{v}))$, the right side is $T\left(\hat{\hat{\pi}}^{M} \circ \hat{\pi}^{M}\right)(\rho(\mathrm{v}))$. The identity $\beta^{M} \circ \bar{\pi}^{M}=\hat{\hat{\pi}}^{M} \circ \hat{\pi}^{M}$ is nothing but (34).

Suppose next that $\hat{\hat{s}} \in \operatorname{Sec}(\hat{\hat{\tau}})$ is $\beta^{\vee}$-related to $\overline{\mathrm{s}} \in \operatorname{Sec}(\bar{\tau})$, i.e. $\hat{\hat{s}}\left(\left[[m]_{N}\right]_{H}\right)=$ $\beta_{[m]_{G}}^{\vee}\left(\overline{\mathrm{S}}\left([m]_{G}\right)\right)$. Then it is easy to see that $\hat{\mathrm{s}}^{I, H} \in \operatorname{Sec}^{I, H}(\hat{\tau})$ will be $\hat{\pi}^{\vee}$-related to $\overline{\mathrm{s}}^{I, G} \in \operatorname{Sec}^{I, G}(\tau)$. Indeed,

$$
\begin{aligned}
\hat{\hat{\mathrm{s}}}^{I, H}\left([m]_{N}\right) & =\left(\hat{\hat{\pi}}_{[m]_{N}}^{\vee}\right)^{-1}\left(\hat{\hat{\mathbf{s}}}\left(\left[[m]_{N}\right]_{H}\right)\right)=\left(\left(\hat{\hat{\pi}}_{[m]_{N}}^{\mathrm{V}}\right)^{-1} \circ \beta_{[m]_{G}}^{\mathrm{V}}\right)\left(\overline{\mathbf{s}}\left([m]_{G}\right)\right) \\
& =\left(\hat{\pi}_{m}^{\mathrm{V}} \circ\left(\bar{\pi}_{m}^{\mathrm{V}}\right)^{-1}\right)\left(\overline{\mathbf{s}}\left([m]_{G}\right)\right)=\left[\overline{\mathbf{s}}^{I, G}(m)\right]_{N},
\end{aligned}
$$

where we made use of (37). The converse is also true: if a $H$-invariant section of $\hat{\tau}$ and a $G$-invariant section of $\tau$ are $\hat{\pi}^{\vee}$-related, then their projections on $\hat{\tau}$ and $\bar{\tau}$, respectively, will be $\beta^{\vee}$-related. With a similar reasoning as in the proof of proposition 4 , we can conclude from the fact that $\hat{\pi}^{\mathrm{V}}$ is a Lie algebroid morphism that $\beta^{\mathrm{V}}$ must also be a Lie algebroid (iso)morphism.

Observe finally that relation (39) is independent of the choice of $m$ and defines a Lie algebra isomorphism $\left(\hat{\pi}^{\vee}\right)^{*}$ between $\operatorname{Sec}^{I, G}(\tau)$ and $\operatorname{Sec}^{I, H}(\hat{\tau})$. Therefore, it is also clear that $\operatorname{Sec}^{I, G}(\tau), \operatorname{Sec}(\bar{\tau}), \operatorname{Sec}^{I, H}(\hat{\tau})$ and $\operatorname{Sec}(\hat{\hat{\tau}})$ are all isomorphic as Lie algebras. 


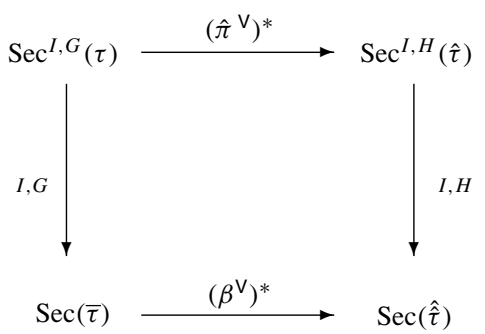

Theorem 2. The dynamics obtained by an iterative reduction (by $N$ and then by $H$ ) is equivalent with the one obtained from a reduction by $G$ directly.

Proof. A similar reasoning as in the proof of the previous proposition shows that $\beta^{\mathrm{W}}$ is admissible with respect to the anchors $\bar{\lambda}$ and $\hat{\hat{\lambda}}$. Thus, $\mathcal{T}^{\beta^{\mathrm{W}}} \beta^{\mathrm{W}}: T^{\bar{\lambda}} \overline{\mathrm{W}} \rightarrow T^{\hat{\hat{\lambda}}} \hat{\hat{\mathrm{W}}}$ is a welldefined isomorphism of vector bundles. The reduction of the dynamics by $G$ gives rise to a section $\bar{\Gamma} \in \operatorname{Sec}\left(\bar{\mu}^{\bar{\lambda}}\right)$, related to the original dynamics $\Gamma \in \operatorname{Sec}\left(\mu^{\lambda}\right)$ by means of (31). Applying $\mathcal{T}^{\beta^{\mathrm{w}}} \beta^{\mathrm{W}}$ on both sides of this relation, and using (13) and relation (38), we find that

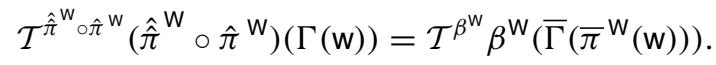

Using the relations between $\Gamma$ and $\hat{\Gamma}$ on the one hand and between $\hat{\Gamma}$ and $\hat{\Gamma}$ on the other, it is easy to see that also

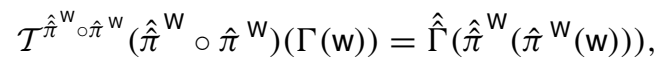

and thus

$$
\mathcal{T}^{\beta^{\mathrm{w}}} \beta^{\mathrm{w}}\left(\bar{\Gamma}\left([\mathrm{w}]_{G}\right)=\hat{\hat{\Gamma}}\left(\beta^{\mathrm{w}}\left([\mathrm{w}]_{G}\right)\right)\right.
$$

or, for the related vector fields $\bar{X}=\bar{\lambda}^{\bar{\mu}}(\bar{\Gamma})$ and $\hat{\hat{X}}=(\hat{\hat{\lambda}})^{\hat{\hat{\mu}}}(\hat{\hat{\Gamma}})$ on $\bar{W}$ and $\hat{\hat{W}}$, respectively,

$$
T \beta^{\mathrm{w}}\left(\bar{X}\left([\mathrm{w}]_{G}\right)\right)=\hat{\hat{X}}\left(\beta^{\mathrm{w}}\left([\mathrm{w}]_{G}\right)\right),
$$

and thus $\hat{\hat{X}}=\beta_{*}^{\mathrm{W}}(\bar{X})$.

Remark that $\mathcal{T}^{\beta^{\mathrm{W}}} \beta^{\mathrm{W}}$ is the restriction to $T^{\lambda} \mathrm{W}$ of $\mathcal{T}^{\beta^{\vee}} \beta^{\mathrm{V}}$. So, for unconstrained systems, i.e. the case where $\mu=\tau$, the projection $\bar{\Gamma} \mapsto \hat{\hat{\Gamma}}$ is really induced by a Lie algebroid isomorphism $\mathcal{T}^{\beta^{\vee}} \beta^{\mathrm{V}}$.

The results above can be applied, for example, to the Lagrange-d'Alembert equations of a non-holonomic system that is invariant under the action of a Lie group $G$ with a nested normal subgroup structure

$$
\{e\} \subset \cdots \subset N_{2} \subset N_{1} \subset G
$$

(with $N_{i+1} \subset N_{i}$ normal subgroups). We have already mentioned that, for each action $\psi^{M}$, the tangent action $\psi^{T M}$ is a Lie algebroid action. Thus, for a $G$-invariant Lagrangian $L \in C^{\infty}(T M)$ and an appropriate constraint distribution $\mathrm{W} \subset T M$, we can perform without further assumptions a successive reduction process. All steps can be interpreted as reducing a Lagrangian system on a subbundle of a Lie algebroid to a system on its quotient. The diagram shows the situation for $\{e\} \subset N_{2} \subset N_{1} \subset G$, with $H_{01}=G / N_{1}, H_{02}=G / N_{2}$ and $H_{12}=N_{1} / N_{2}$. All vertical arrows are Lie algebra isomorphisms, all horizontal arrows are 
injections. At each stage in the reduction process, the dynamics is defined on a subbundle of the Lie algebroid at the end of the line.

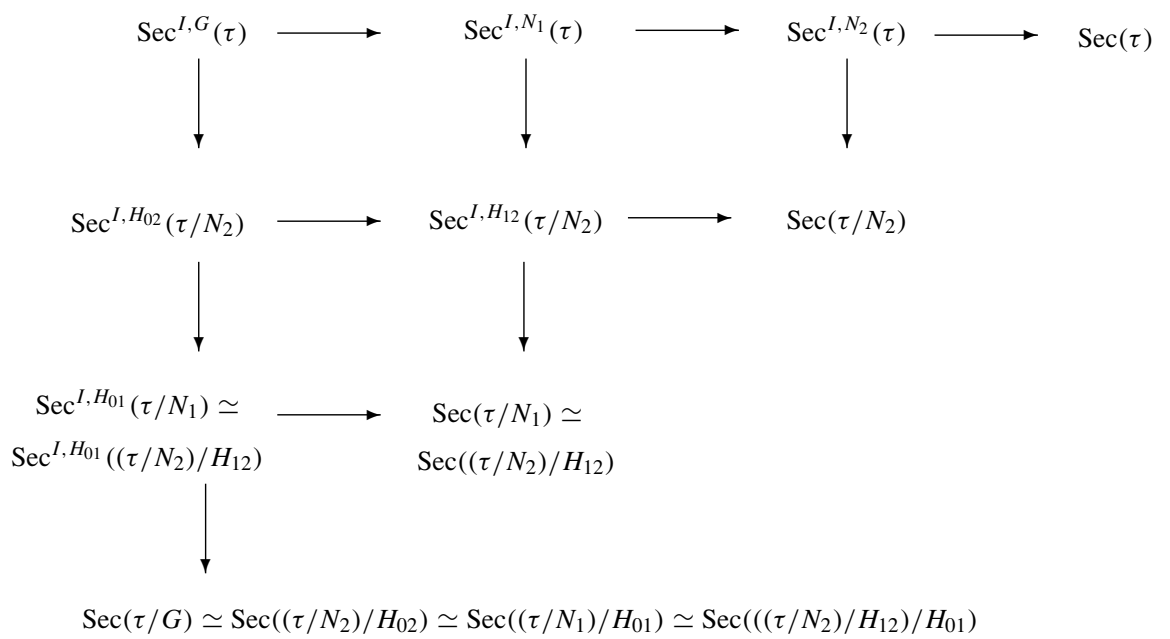

\section{Coordinate expressions and examples}

Before we describe some examples in detail, it may be instructive to provide a local version of what preceded. Assume that a local coordinate chart $\left(U,\left(x^{i}\right)\right)$ of $M$ is given and that $\left\{\mathrm{e}_{a}\right\}$ and $\left\{f_{A}\right\}$ are bases of, respectively, $\operatorname{Sec}(\tau)$ and $\operatorname{Sec}(\mu)$. Fibre coordinates on $\mathrm{V}$ and $\mathrm{W}$, with respect to these bases, will be denoted by $v^{a}$ and $w^{A}$ and $i_{A}^{a}$ for the components of the injection $i: \mathrm{W} \rightarrow \mathrm{V}$. The structure functions of the Lie algebroid structure on $\tau$ are given by $\rho_{a}^{i}$ and $C_{a b}^{c}$, say, and finally $\lambda_{A}^{i}=i_{A}^{a} \rho_{a}^{i}$.

Let $L$ be a regular Lagrangian on $\mathrm{V}$. The dynamics is represented by a section $\Gamma$ of the prolongation bundle $\mu^{\lambda}$. Recall that projectable sections $\mathcal{Z}$ of the prolongation bundle $\mu^{\lambda}$ consist of a pair $(r, X)$, with $r \in \operatorname{Sec}(\tau)$ and $X \in \mathcal{X}(\mathrm{W})$. A natural choice for a basis for $\operatorname{Sec}\left(\mu^{\lambda}\right)$ is $\left\{\mathcal{X}_{A}, \mathcal{V}_{A}\right\}$, with

$\mathcal{X}_{A}(\mathrm{w})=\left(\mathrm{f}_{A}(m),\left.\lambda_{A}^{i} \frac{\partial}{\partial x^{i}}\right|_{\mathrm{w}}\right) \quad$ and $\quad \mathcal{V}_{A}(\mathrm{w})=\left(0(m),\left.\frac{\partial}{\partial \mathrm{w}^{A}}\right|_{\mathrm{w}}\right)$,

where $\mathrm{w} \in \mathrm{W}$ and $m=\mu(\mathrm{w})$. The solution $\Gamma$ of (21) is of the form

$$
\Gamma=\mathrm{w}^{A} \mathcal{X}_{A}+f^{A}\left(x^{i}, \mathrm{w}^{B}\right) \mathcal{V}_{A},
$$

for certain coefficients $f^{A} \in C^{\infty}(\mathrm{W})$. According to definition 3, the Lagrangian equations under consideration are then the differential equations for the integral curves of the corresponding vector field

$$
\lambda^{\mu}(\Gamma)=\mathrm{w}^{A} \lambda_{A}^{i} \frac{\partial}{\partial x^{i}}+f^{A} \frac{\partial}{\partial \mathrm{w}^{A}} \in \mathcal{X}(\mathrm{W}) .
$$

In [12] (equation (4)), we have shown that these equations can be written in the form

$$
\left\{\begin{array}{l}
\dot{x}^{i}=\rho_{a}^{i}(x) \mathrm{v}^{a}, \\
\mathrm{v}^{a}=i_{A}^{a}(x) \mathrm{w}^{A}, \\
i_{A}^{a}\left(\frac{\mathrm{d}}{\mathrm{d} t}\left(\frac{\partial L}{\partial \mathrm{v}^{a}}\right)\right)=i_{A}^{a}\left(\rho_{a}^{i} \frac{\partial L}{\partial x^{i}}-C_{a b}^{c} \mathrm{v}^{b} \frac{\partial L}{\partial \mathrm{v}^{c}}\right) .
\end{array}\right.
$$


Let $G$ now be a Lie group acting on the Lie algebroid $\tau$ and assume that our Lagrangian is $G$-reducible. Let $\bar{X} \times G\left(\bar{X} \subset \mathbb{R}^{n}, n=\operatorname{dim} \bar{M}\right)$ be a local trivialization of the principal fibre bundle $\bar{\pi}^{M}: M \rightarrow \bar{M}$. If $\bar{x}^{k}$ are coordinates on $\bar{X}$, then $\bar{\pi}^{M}$ is locally $x^{i}=\left(\bar{x}^{k}, g^{l}\right) \mapsto \bar{x}^{k}$. In this set of coordinates, $\psi^{M}$ is simply $(h,(\bar{x}, g)) \rightarrow(\bar{x}, h g)$.

In order to give an idea of how reduction works in coordinates, we choose a basis $\left\{\mathrm{e}_{a}\right\}$ of $\operatorname{Sec}(\tau)$ which is made up of invariant sections. In such a coordinate system $\psi^{\vee}: G \times \mathrm{V} \rightarrow \mathrm{V}$ takes the form $\left(h, \mathrm{v}^{a} \mathrm{e}_{a}(\bar{x}, g)\right) \rightarrow \mathrm{v}^{a} \mathrm{e}_{a}(\bar{x}, h g)$. Moreover, the invariant basis $\left\{\mathrm{e}_{a}\right\}$ projects to a basis of the quotient Lie algebroid $\bar{\tau}$. It will also be convenient to denote this new basis by $\left\{\mathrm{e}_{a}\right\}$. The only difference between $\mathrm{e}_{a}$ as a section of $\tau$ on the one hand and as a section of $\bar{\tau}$ on the other is that, in the first case, it can be evaluated at a point of $M$, while, in the second, it should be interpreted as acting on elements of $\bar{M}$. Taking this identification into account, the projection $\bar{\pi}^{\vee}$ is simply $\mathrm{v}^{a} \mathrm{e}_{a}(\bar{x}, g) \rightarrow \mathrm{v}^{a} \mathrm{e}_{a}(\bar{x})$.

Theorem 1 showed that the reduced system can be identified with a section $\bar{\Gamma}$ of the prolongation bundle $\bar{\mu}^{\bar{\lambda}}$. A coordinate expression for this system, similar to (42), is (the meaning of the notations being obvious)

$$
\left\{\begin{array}{l}
\dot{\bar{x}}^{k}=\bar{\rho}_{a}^{k}(\bar{x}) \mathrm{v}^{a}, \\
\mathrm{v}^{a}=\bar{i}_{A}^{a}(\bar{x}) \mathrm{w}^{A}, \\
\bar{i}_{A}^{a}\left(\frac{\mathrm{d}}{\mathrm{d} t}\left(\frac{\partial \bar{L}}{\partial \mathrm{v}^{a}}\right)\right)=\bar{i}_{A}^{a}\left(\bar{\rho}_{a}^{k} \frac{\partial \bar{L}}{\partial \bar{x}^{k}}-\bar{C}_{a b}^{c} \mathrm{v}^{b} \frac{\partial \bar{L}}{\partial \mathrm{v}^{c}}\right) .
\end{array}\right.
$$

Since the invariant sections form a Lie sub-algebra, it is clear that the structure functions $C_{a b}^{c}(\bar{x}, g)$ with respect to $\left\{\mathrm{e}_{a}\right\}$ are invariant functions, and thus are independent of the coordinates $g^{l}$. From (7), we can moreover conclude that $\bar{C}_{a b}^{c}(\bar{x})=C_{a b}^{c}(\bar{x}, g)$. Likewise (in the above identification), the quotient anchor map is $\bar{\rho}: \mathrm{v}^{a} \mathrm{e}_{a}(\bar{x}) \mapsto \bar{\rho}_{a}^{k} \frac{\partial}{\partial \bar{x}^{k}}(\bar{x})$, where $\bar{\rho}_{a}^{k}(\bar{x})=\rho_{a}^{k}(\bar{x}, g)$ is the first set of the components of $\rho$. If we further take $\left\{\mathrm{f}_{A}\right\}$ to be a $G$-invariant basis of $\operatorname{Sec}(\mu)$ (and if we identify again these sections with their projections on $\operatorname{Sec}(\bar{\mu})$ ), then, due to (23), the induced injection $\bar{i}: \overline{\mathrm{W}} \rightarrow \overline{\mathrm{V}}$ is simply $\mathrm{w}^{A} \mathrm{f}_{A} \mapsto \bar{i}_{A}^{a} \mathrm{w}^{A} \overline{\mathrm{e}}_{A}$, where $\bar{i}_{A}^{a}(\bar{x})=i_{A}^{a}(\bar{x}, g)$. To ease the notations, we will from now on remove all the bars from the structure functions on $\bar{\tau}$ and $\bar{\mu}$, in accordance with earlier made identifications. Finally, the invariance of the Lagrangian can be expressed as $L\left(\bar{x}^{k},(h g)^{l}, \mathrm{v}^{a}\right)=L\left(\bar{x}^{k},(g)^{l}, \mathrm{v}^{a}\right), \forall h \in G$, which means that $\frac{\partial L}{\partial g^{l}}=0$. In particular, in the first term of the right-hand side of the last of equations (42) only derivatives of the form $\frac{\partial L}{\partial \bar{x}^{k}}$ will remain. From all this, it is obvious that (42) projects on (43).

We can define also a basis $\left\{\overline{\mathcal{X}}_{A}, \overline{\mathcal{V}}_{A}\right\}$ for $\operatorname{Sec}\left(\bar{\mu}^{\bar{\lambda}}\right)$, similar to the basis (40) of $\operatorname{Sec}\left(\mu^{\lambda}\right)$. However, by the conventions we adopted for denoting coordinates, bases and structure functions, these sections can formally be identified with the sections $\left\{\mathcal{X}_{A}, \mathcal{V}_{A}\right\}$ and we will again make no notationial distinction. With this identification in mind, the reduced dynamics $\bar{\Gamma} \in \operatorname{Sec}\left(\bar{\mu}^{\bar{\lambda}}\right)$ looks formally the same as expression (41) for $\Gamma \in \operatorname{Sec}\left(\mu^{\lambda}\right)$. The corresponding vector field is, however,

$$
\bar{\lambda}^{\bar{\mu}}(\bar{\Gamma})=\mathrm{w}^{A} \lambda_{A}^{k} \frac{\partial}{\partial \bar{x}^{k}}+f^{A} \frac{\partial}{\partial \mathrm{w}^{A}} \in \mathcal{X}(\overline{\mathrm{W}}) .
$$

Next, we discuss some examples where an iterative reduction process applies.

\subsection{The non-holonomically constrained particle}

In this example $M=\mathbb{R}^{3}$ and $\mathrm{V}=T M$ (with its standard Lie algebroid structure). The system has a Lagrangian $L(x, y, z, \dot{x}, \dot{y}, \dot{z})=\frac{1}{2}\left(\dot{x}^{2}+\dot{y}^{2}+\dot{z}^{2}\right)$ and is subjected to a constraint, 
characterized by a distribution $\mathrm{W} \subset T M$ with equation $\dot{z}=y \dot{x}$ (see also [1]). The injection $i$ is then given by $(x, y, z, \dot{x}, \dot{y}) \mapsto(x, y, z, \dot{x}, \dot{y}, y \dot{x})$, or, for the corresponding basis $\left\{\mathrm{f}_{1}, \mathrm{f}_{2}\right\}$ of $\operatorname{Sec}(\mu), i\left(\mathrm{f}_{1}\right)=X_{1}=\frac{\partial}{\partial x}+y \frac{\partial}{\partial z}$ and $i\left(\mathrm{f}_{2}\right)=X_{2}=\frac{\partial}{\partial y}$. The basis (40) is here given by the sections $\mathcal{X}_{1}=\left(\mathrm{f}_{1}, X_{1}\right), \mathcal{X}_{2}=\left(\mathrm{f}_{2}, X_{2}\right), \mathcal{V}_{1}=\left(0, \frac{\partial}{\partial \dot{x}}\right)$ and $\mathcal{V}_{2}=\left(0, \frac{\partial}{\partial \dot{y}}\right)$. An easy calculation (based on, e.g., the coordinate expressions (42)) shows that the dynamics $\Gamma \in \operatorname{Sec}\left(\mu^{\lambda}\right)$ of this example is given by

$$
\Gamma=\dot{x} \mathcal{X}_{1}+\dot{y} \mathcal{X}_{2}-\frac{y}{1+y^{2}} \dot{x} \dot{y} \mathcal{V}_{1}
$$

or by the corresponding vector field

$$
\lambda^{\mu}(\Gamma)=\dot{x} \frac{\partial}{\partial x}+\dot{y} \frac{\partial}{\partial y}+y \dot{x} \frac{\partial}{\partial z}-\frac{y}{1+y^{2}} \dot{x} \dot{y} \frac{\partial}{\partial \dot{x}} \in \mathcal{X}(\mathrm{W}) .
$$

Consider now, first, the action of $G=\mathbb{R}^{2}$ on $M=\mathbb{R}^{3}$, given by

$$
\psi^{\vee}:((r, s) ;(x, y, z, \dot{x}, \dot{y}, \dot{z})) \mapsto(x+r, y, z+s, \dot{x}, \dot{y}, \dot{z}) .
$$

Then, clearly the restriction of $\psi^{\vee}$ to $\mathrm{W}$ is a constrained Lie algebroid action for this system. The projections on the quotient manifolds are in this case $\bar{\pi}^{M}:(x, y, z) \mapsto y, \bar{\pi}^{\mathrm{V}}$ : $(x, y, z, \dot{x}, \dot{y}, \dot{z}) \mapsto(y, \dot{x}, \dot{y}, \dot{z})$ and $\bar{\pi}^{\mathrm{W}}:(x, y, z, \dot{x}, \dot{y}) \mapsto(y, \dot{x}, \dot{y})$, while the quotient injection $\bar{i}$ is $(y, \dot{x}, \dot{y}) \mapsto(y, \dot{x}, \dot{y}, y \dot{x})$. The bases $\left\{\mathrm{f}_{1}, \mathrm{f}_{2}\right\}$ and $\left\{\frac{\partial}{\partial x}, \frac{\partial}{\partial y}, \frac{\partial}{\partial z}\right\}$ for $\mu$ and $\tau$ consist clearly only of invariant sections. As has been said above, the reduced dynamics $\bar{\Gamma} \in \operatorname{Sec}\left(\bar{\mu}^{\bar{\lambda}}\right)$ looks formally the same as expression (44) for $\Gamma \in \operatorname{Sec}\left(\mu^{\lambda}\right)$. The vector field $\bar{\lambda}^{\bar{\mu}}(\bar{\Gamma})$ is, however,

$$
\dot{y} \frac{\partial}{\partial y}-\frac{y}{1+y^{2}} \dot{x} \dot{y} \frac{\partial}{\partial \dot{x}} \in \mathcal{X}(\overline{\mathrm{W}}) .
$$

Instead of making a reduction by the whole $G=\mathbb{R}^{2}$, we can work also in two stages: one can first look at the restriction of the action $\psi^{\vee}$ to its (normal) subgroup $N=\mathbb{R}=\mathbb{R} \times\{0\}$. Then, denoting the reduction process now by ${ }^{\wedge}$, the reduced injection is $\hat{i}:(y, z, \dot{x}, \dot{y}) \mapsto(y, z, \dot{x}, \dot{y}, y \dot{x})$ and, again, $\hat{\Gamma} \in \operatorname{Sec}\left(\hat{\mu}^{\hat{\lambda}}\right)$ will take the same form as $\Gamma$ (leaving aside the fact that $\hat{\Gamma}$ acts on elements $(y, z, \dot{x}, \dot{y}) \in \hat{\mathrm{W}})$. The vector field of interest is now

$$
\hat{\lambda}^{\hat{\mu}}(\hat{\Gamma})=\dot{y} \frac{\partial}{\partial y}+y \dot{x} \frac{\partial}{\partial z}-\frac{y}{1+y^{2}} \dot{x} \dot{y} \frac{\partial}{\partial \dot{x}} \in \mathcal{X}(\hat{\mathrm{W}}) .
$$

For the above system, the Lagrangian is $\hat{L}(z, y, \dot{x}, \dot{y}, \dot{z})=\frac{1}{2}\left(\dot{x}^{2}+\dot{y}^{2}+\dot{z}^{2}\right)$, while the constraint is still $\dot{z}-y \dot{x}=0$. Since both are invariant under the $\mathbb{R}$-action

$$
\psi^{\hat{V}}:(s ;(y, z, \dot{x}, \dot{y}, \dot{z})) \mapsto(y, z+s, \dot{x}, \dot{y}, \dot{z}),
$$

a second reduction can be performed to obtain $\hat{\Gamma} \in \operatorname{Sec}\left(\hat{\hat{\mu}}^{\hat{\hat{\mu}}}\right)$. Since, again, $\hat{\hat{\Gamma}}$ takes the same form as $\Gamma$ and thus also as $\bar{\Gamma}$ (under appropriate identifications), it becomes clear that reduction by two stages for this non-holonomically constrained particle is equivalent with direct reduction. Likewise, $\hat{\hat{\lambda}}^{\hat{\hat{\mu}}}(\hat{\hat{\Gamma}}) \in \mathcal{X}(\hat{\hat{W}})$ can formally be identified with expression (45) for $\bar{\lambda}^{\bar{\mu}}(\bar{\Gamma})$.

\subsection{The falling rolling disk}

In this example we consider a homogeneous disk rolling over a horizontal plane. The constraint is that the disk rolls without slipping. We will use the same notations as in 
[1, p 21]: $(x, y)$ denote the coordinates of the contact point in the plane on which the disk rolls, and $\theta, \phi$ and $\psi$ are the angle between the plane and the vertical axis, the 'heading angle' of the disk, and the 'self-rotation' angle of the disk, respectively. In this example, $M=S E(2) \times S O(2) \times S O(2), \mathrm{V}=T M$ and the Lagrangian is

$$
\begin{aligned}
L=\frac{m}{2}\left[(\zeta-R \dot{\phi} \sin \theta)^{2}+\eta^{2} \sin ^{2} \theta+(\eta \cos \theta+R \dot{\theta})^{2}\right] \\
+\frac{1}{2}\left[J\left(\dot{\theta}^{2}+\dot{\phi}^{2} \cos ^{2} \theta\right)+I(\dot{\phi} \sin \theta+\dot{\psi})^{2}\right]-m g R \cos \theta
\end{aligned}
$$

with $\zeta=\dot{x} \cos \phi+\dot{y} \sin \phi$ and $\eta=-\dot{x} \sin \phi+\dot{y} \cos \phi$ and $I$ and $J$ are inertia constants. The constraints are given by the equations

$$
\left\{\begin{array}{l}
\dot{x}=-\dot{\psi} R \cos \phi, \\
\dot{y}=-\dot{\psi} R \sin \phi .
\end{array}\right.
$$

The falling rolling disk is invariant under the action of the group $G=S E(2) \times S O(2)$ on $M$

$$
\begin{gathered}
\psi_{G}^{M}:(a, b, \alpha, \beta) \times(x, y, \phi, \psi, \theta) \mapsto(x \cos \alpha-y \sin \alpha+a, x \sin \alpha \\
+y \cos \alpha+b, \phi+\alpha, \psi+\beta, \theta)
\end{gathered}
$$

and its induced tangent action

$$
\begin{aligned}
\psi_{G}^{\vee}:(a, b, \alpha, \beta) & \times(x, y, \phi, \psi, \theta, \dot{x}, \dot{y}, \dot{\phi}, \dot{\psi}, \dot{\theta}) \\
& \mapsto\left(\psi_{G}^{M}(x, y, \phi, \psi, \theta), \dot{x} \cos \alpha-\dot{y} \sin \alpha, \dot{x} \sin \alpha+\dot{y} \cos \alpha, \dot{\phi}, \dot{\psi}, \dot{\theta}\right) .
\end{aligned}
$$

$\psi_{G}^{\vee}$ is by construction a Lie algebroid action for the Lie algebroid $T M$ and it restricts to a constrained Lie algebroid action on the subbundle W. The basis $\left\{\mathfrak{f}_{\theta}, f_{\phi}, f_{3}\right\}$ of $\operatorname{Sec}(\mu)$ with

$$
i\left(\mathfrak{f}_{\theta}\right)=\frac{\partial}{\partial \theta}, \quad i\left(\mathfrak{f}_{\phi}\right)=\frac{\partial}{\partial \phi}, \quad i\left(\mathrm{f}_{3}\right)=\frac{\partial}{\partial \psi}-R \cos \phi \frac{\partial}{\partial x}-R \sin \phi \frac{\partial}{\partial y}
$$

is invariant under the restricted action $\psi_{G}^{\mathrm{W}}$. Fibre coordinates on $\mathrm{W}$ will be denoted by $\left(\dot{\theta}, \dot{\phi}, \mathrm{w}^{3}\right)$. The natural basis $\left\{\frac{\partial}{\partial x}, \frac{\partial}{\partial y}, \frac{\partial}{\partial \theta}, \frac{\partial}{\partial \phi}, \frac{\partial}{\partial \psi}\right\}$ on $\mathrm{V}=T M$ is, however, not invariant under $\psi_{G}^{\vee}$. An invariant basis $\left\{\mathrm{e}_{a}\right\}$ on $T M$ is, e.g.,

$$
\left\{\mathrm{e}_{\zeta}=\cos \phi \frac{\partial}{\partial x}+\sin \phi \frac{\partial}{\partial y}, \mathrm{e}_{\eta}=-\sin \phi \frac{\partial}{\partial x}+\cos \phi \frac{\partial}{\partial y}, \mathrm{e}_{\theta}=\frac{\partial}{\partial \theta}, \mathrm{e}_{\phi}=\frac{\partial}{\partial \phi}, \mathrm{e}_{\psi}=\frac{\partial}{\partial \psi}\right\},
$$

and we will from now on use induced coordinates $(x, y, \theta, \phi, \psi ; \zeta, \eta, \dot{\theta}, \dot{\phi}, \dot{\psi})$ on $T M$, where $\zeta$ and $\eta$ are as above. In the new coordinates the constraints can be written as $\zeta=-R \dot{\psi}$ and $\eta=0$. This means that the only non-vanishing components of the injection are $i_{\theta}^{\theta}=1, i_{\phi}^{\phi}=1, i_{3}^{\psi}=1$ and $i_{3}^{\zeta}=-R$. The change of basis has the side effect that the structure functions of the Lie algebroid need to be recalculated. Of course, $\rho$ is still the identity, but, where in the standard basis the structure functions $C_{a b}^{c}$ of the standard Lie algebroid all vanish, there arise non-vanishing brackets for the new basis sections. In particular, $\left[\mathrm{e}_{\zeta}, \mathrm{e}_{\phi}\right]=-\mathrm{e}_{\eta}$ and $\left[\mathrm{e}_{\eta}, \mathrm{e}_{\phi}\right]=\mathrm{e}_{\zeta}$, so the non-vanishing structure functions are $C_{\zeta \phi}^{\eta}=-1$ and $C_{\eta \phi}^{\zeta}=1$. An explicit expression for the Lagrangian equations now follows easily from (42). To proceed further, it suffices to know that the section $\Gamma$ is of the form

$$
\Gamma=\dot{\theta} \mathcal{X}_{\theta}+\dot{\phi} \mathcal{X}_{\phi}+w^{3} \mathcal{X}_{3}+f^{\theta} \mathcal{V}_{\theta}+f^{\phi} \mathcal{V}_{\phi}+f^{3} \mathcal{V}_{3}
$$

and that the associated vector field is

$\lambda^{\mu}(\Gamma)=\dot{\theta} \frac{\partial}{\partial \theta}+\dot{\phi} \frac{\partial}{\partial \phi}+w^{3}\left(\frac{\partial}{\partial \psi}-R \cos \phi \frac{\partial}{\partial x}-R \sin \phi \frac{\partial}{\partial y}\right)+f^{\theta} \frac{\partial}{\partial \dot{\theta}}+f^{\phi} \frac{\partial}{\partial \dot{\phi}}+f^{3} \frac{\partial}{\partial \dot{w}^{3}}$. 
Let us look at the reduced system now: $\theta$ can be regarded as the only coordinate on $\bar{M}$, while, as before, we denote coordinates on $\overline{\mathrm{V}}$ as $(\theta, \zeta, \eta, \dot{\theta}, \dot{\phi}, \dot{\psi})$. The reduced Lagrangian looks exactly like (46), only now it should be interpreted as a function in the variables of $\bar{V}$ alone. Also the constraints remain simply $\zeta=-R \dot{\psi}$ and $\eta=0$. We have seen above that, within the earlier discussed identifications, all reduced sections have the same form as $\Gamma$. The $G$-reduced vector field is here

$$
\bar{\lambda}^{\bar{\mu}}(\bar{\Gamma})=\dot{\theta} \frac{\partial}{\partial \theta}+f^{\theta} \frac{\partial}{\partial \dot{\theta}}+f^{\phi} \frac{\partial}{\partial \dot{\phi}}+f^{3} \frac{\partial}{\partial \dot{\mathrm{w}}^{3}} .
$$

An iterative reduction process now becomes available. In fact, we have a nested subgroup structure $\mathbb{R}^{2} \subset S E(2) \subset S E(2) \times S O(2)$, so we can perform reduction in three steps: the corresponding groups for the diagram at the end of the previous section are $H_{01}=S O(2), H_{02}=S O(2) \times S O(2)$ and $H_{12}=S O(2)$. We will limit ourselves, however, to a reduction in two steps. The first step will be reduction by the group $N_{1}=S E(2)$, whose restricted action on $M$ is

$$
\begin{aligned}
\psi_{N_{1}}^{M}:(a, b, \alpha) & \times(x, y, \phi, \psi, \theta) \mapsto(x \cos \alpha-y \sin \alpha+a, x \sin \alpha \\
& +y \cos \alpha+b, \phi+\alpha, \psi, \theta)
\end{aligned}
$$

The earlier discussed bases are also invariant under the tangent lift of $\psi_{N_{1}}^{M}$, but the coordinates on $\hat{M}$ are now $(\theta, \phi)$. The Lagrangian $\hat{L}$ looks formally the same as expression (47) again, but it is to be interpreted as a function of the variables $(\theta, \phi, \zeta, \eta, \dot{\theta}, \dot{\phi}, \dot{\psi})$. A similar observation is valid for the reduced constraint. The vector field of interest after the reduction by $N_{1}$ is

$$
\hat{\lambda}^{\hat{\mu}}(\hat{\Gamma})=\dot{\theta} \frac{\partial}{\partial \theta}+\dot{\phi} \frac{\partial}{\partial \phi}+f^{\theta} \frac{\partial}{\partial \dot{\theta}}+f^{\phi} \frac{\partial}{\partial \dot{\phi}}+f^{3} \frac{\partial}{\partial \dot{\mathrm{w}}^{3}} .
$$

The symmetry group $H_{1}=G / N_{1}$ for the second stage is $S O(2)$. Since both the Lagrangian $\hat{L}$ and the reduced constraint are still independent of the coordinate $\phi$, i.e., invariant under the action

$$
\psi_{H_{1}}^{\hat{M}}: \beta \times(\psi, \theta) \mapsto(\psi+\beta, \theta),
$$

a second reduction can be performed. The coordinate of $\hat{\hat{M}}$ is again $\theta$ and also the corresponding vector field $\hat{\hat{\lambda}}^{\hat{\hat{\mu}}}(\hat{\hat{\Gamma}})$ can be identified with expression (48) for $\bar{\lambda}^{\bar{\mu}}(\bar{\Gamma})$.

\section{Acknowledgment}

I am indebted to Willy Sarlet for useful discussions.

\section{References}

[1] Bloch A M 2003 Nonholonomic mechanics and control (Interdisciplinary Applied Mathematics vol 24) (New York: Springer)

[2] Cendra H, Marsden J E and Ratiu T S 2001 Lagrangian Reduction by Stages (Mem. Am. Math. Soc. vol 152)

[3] Cendra H, Marsden J E and Ratiu T S 2001 Geometric mechanics, Lagrangian reduction, and nonholonomic systems Mathematics Unlimited-2001 and Beyond ed B Engquist and W Schmid (Berlin: Springer) pp 221-73

[4] Cortés J and Martínez E 2004 Mechanical control systems on Lie algebroids IMA J. Math. Control Inf. 21 457-92

[5] de León M, Marrero J C and Martínez E 2005 Lagrangian submanifolds and dynamics on Lie algebroids J. Phys. A: Math. Gen. 38 R241-308

[6] Higgins P J and Mackenzie K 1990 Algebraic constructions in the category of lie algebroids J. Algebra 129 194-230 
[7] Mackenzie K 1987 Lie Groupoids and Lie Algebroids in Differential Geometry (London Math. Soc. Lect. Notes Series vol 124) (Cambridge: Cambridge University Press)

[8] Martínez E 2001 Lagrangian mechanics on lie algebroids Acta. Appl. Math. 67 295-320

[9] Martínez E 2004 Classical field theory on Lie algebroids: multisymplectic formalism Preprint math.DG/0411352

[10] Martínez E, Mestdag T and Sarlet W 2002 Lie algebroid structures and Lagrangian systems on affine bundles J. Geom. Phys. 44 70-95

[11] Mestdag T 2005 A Lie algebroid approach to Lagrangian systems with symmetry Diff. Geom. and its Appl. (Proc. Conf. Prague, Aug 30-Sep 3 pp 525-37

[12] Mestdag T and Langerock B 2005 A lie algebroid framework for non-holonomic systems J. Phys. A: Math. Gen. 38 1097-111

[13] Weinstein A 1996 Lagrangian mechanics and groupoids Mechanics Day (Waterloo, ON, 1992) (Providence, RI: American Mathematical Society) pp 207-31 (Fields Inst. Commun. 7) 